\title{
Assessment of cloth masks ability to limit Covid-19 particles spread: a systematic review
}

\author{
Mahshid Ataei $^{1,2} \cdot$ Farshad M. Shirazi $^{3} \cdot$ Samaneh Nakhaee ${ }^{1} \cdot$ Mohammad Abdollahi $^{2} \cdot$ Omid Mehrpour $^{1,4}(\mathbb{C}$
}

Received: 14 April 2021 / Accepted: 28 September 2021 / Published online: 23 October 2021

(c) The Author(s), under exclusive licence to Springer-Verlag GmbH Germany, part of Springer Nature 2021

\begin{abstract}
After the spread of Covid 19 worldwide, the use of cloth masks increased significantly due to a shortage of medical masks. Meanwhile, there were different opinions about the effectiveness of these masks and, so far, no study has been done to find the best fabric masks. This study reviews and summarizes all studies related to fabric masks' effectiveness and various fabrics against coronavirus. This systematic review is based on PRISMA rules. Two researchers separately examined three databases: PubMed, Scopus, and Web of Science. Laboratory and clinical studies were included. After extracting the articles, their quality was assessed with the Joanna Briggs Institute (JBI) tool. In addition to efficacy, other factors, including the penetration of masks, pressure drop, and quality factor, were examined to select the best fabrics. Of the 42 studies selected, 39 were laboratory studies, and 3 were clinical studies. Among the various fabrics examined, cotton quilt 120 thread per inch (TPI), copy paper (bonded), hybrid of cotton with chiffon/ silk, and flannel filtration were found to have over $90 \%$ effectiveness in the particle size range of Covid-19. The results and comparison of different factors (pressure drop, filtration efficacy, penetration, filtration quality, and fit factor have been evaluated) showed that among different fabrics, hybrid masks, 2-layered cotton quilt, 2-layered $100 \%$ cotton, cotton flannel, and hairy tea towel + fleece sweater had the best performance. Clinical studies have not explicitly examined cloth masks' effectiveness in Covid-19, so the effectiveness of these types of masks for Covid 19 is questionable, and more studies are needed.
\end{abstract}

Keywords Mask $\cdot$ Covid-19 $\cdot$ Prevention

\section{Introduction}

In early December 2019, in Wuhan's city in Hubei province, China, many people caught pneumonia. After a while, the cause of this cluster of diseases became known, which was a novel virus from the coronavirus's family. Later, the disease was named Covid-19, caused by the SARS-Cov 2 virus (Chinazzi et al. 2020). This new coronavirus had a $79 \%$ sequence similarity to SARS-Cov, which caused a significant outbreak in 2002-2003 (Lake 2020). It did not take long that Covid-19 disease became a pandemic and a global concern that killed more than 1.3 million people up to November 2020, and in most countries, the rate of Covid-19 confirmed cases was rapidly growing, according to World Health Organization (WHO) (Anonymous). Because the SARS-Cov 2 virus is so contagious and due to the lockdown removal, everyone needs to take various preventive measures, including washing their hands regularly, using various protective equipment like gloves, gowns, masks, observing social distance,

4 Mel and Enid Zuckerman College of Public Health, University of Arizona, Tucson, AZ, USA 
quarantining infected and suspected people to Covid-19 disease (Santos et al. 2020; Sunjaya and Morawska 2020). One type of mask is cloth masks, which are made of different materials and designs. These different materials and designs affect the mask's filtration efficacy (FE) (Howard et al. 2020). There are different types of fabric masks, of which we can mention knitted (interlocking fiber loops), woven (crossing threads are known as warp and weave), or felted (compressed, disorganized fibers). Fabric masks can partially block the transmission of respiratory droplets from people who wear them compared to those who do not wear masks. This blocking effect increases by increasing the number of fabric layers (Clase et al. 2020). Only some fabric masks and reusable respirators can be disinfected and reused among different masks without changing the filtration effectiveness (Bhattacharjee et al. 2020). Wearing cloth masks will significantly affect disease control because it can significantly control asymptomatic patients who move freely and speaking, sneezing, or cough. Viral shedding of patients with Covid-19 is higher in the time of symptom onset and before the symptom onset (Santos et al. 2020). Wiersinga et al. showed that asymptomatic carriers transmit the virus to others at a rate of $48-62 \%$ (Wiersinga et al. 2020). Therefore, cloth masks will have an advantageous effect in reducing disease transmission, especially from asymptomatic carriers. According to this, two strategies are suggested:

1) Health care practitioners)HCPs(: For Health care workers, WHO recommended that they should use medical masks and respirators (Organization 2020a, b). Macintyre's research also showed that the HCPs Chughtai AA, Seale H, Macintyre CRwho used cloth masks had a higher risk of getting influenza-like illness than those who used medical masks (MacIntyre et al. 2015b).

2) General population: To maintain medical masks and respirators for the HCPs, the CDC recommends using cloth masks for general use that are very economical and accessible (Sunjaya and Morawska 2020). WHO was initially against the use of cloth masks, so that on 19 March, WHO claimed that "Cloth (e.g., cotton or gauze) masks are not recommended under any circumstances" but, later changed its mind and on 5 June, WHO advised decision-makers to recommend all people wear masks (Clase et al. 2020). Many countries recommended the use of cloth masks for the general population based on their low price, availability, and at the same time, effectiveness. On the other hand, due to the lack of medical masks and respirators, these masks are better kept for the HCPs (Godoy et al. 2020).

Despite the extensive use of cloth masks, few studies conducted a review on their virus-blocking efficacy and summarized such studies. In the present study, we aimed to compare these masks with each other via reviewing all studies related to fabric masks' effectiveness for Covid-19.

\section{Methodology}

This systematic review follows the Preferred Reporting Items for Systematic Reviews and Meta-Analyses (PRISMA) instructions (Moher et al. 2015). The PECO research strategy (Scells et al. 2017) was used in this study containing the following information: $\mathrm{P}=$ droplet and/or aerosol dispersion contamination;

$\mathrm{E}=$ homemade and/or commercial cloth masks; $\mathrm{C}=$ different cloth masks materials.

Outcome $=$ cloth masks efficiency in reducing the transmission of contaminated droplets and aerosols through laboratory and clinical tests. We used medical subject heading (MESH) terms and combined the keywords in the title and abstract (cloth mask, fabric mask, textile mask, homemade mask, cotton mask, Covid-19, SARA-Cov-2, n-Cov-2019) while searching the main international databases, including PubMed, Scopus, Web of Science. Two researchers searched the databases mentioned above up to 5 January 2021 independently. Examples of PubMed search queries using MeSH Terms and the free-text words were as follows:

(((homemade mask*[Title/Abstract]) OR (textile mask*[Title/Abstract]) OR (((cloth mask*[Title/Abstract]) OR (fabric mask*[Title/Abstract])cotton mask*[Title/ Abstract]) OR (gauze mask*[Title/Abstract])) AND ((Covid-19[Title/Abstract]) OR (COVID-19[Title/Abstract]) OR(cloth mask[MeSH Terms]) OR (fabric mask[MeSH Terms]) OR (textile mask[MeSH Terms]) OR (homemade mask[MeSH Terms]) OR (cotton mask[MeSH Terms]) OR (gauze mask[MeSH Terms]) OR (Covid 19[Title/Abstract]) OR (SARS-CoV-2[Title/Abstract]) OR (SARS-Cov2[Title/Abstract]) OR (severe acute respiratory syndrome coronavirus 2[Title/Abstract]) OR (ncov[Title/Abstract]) OR (2019-nCoV[Title/Abstract]) OR (COVID 19[Title/ Abstract]) OR (COVID-19 Virus[Title/Abstract]) OR (Coronavirus Disease 2019[Title/Abstract]) OR (SARS Coronavirus 2[Title/Abstract]OR (Coronavirus Disease-19[Title/ Abstract]), OR (2019 Novel Coronavirus[Title/Abstract])))

\section{Eligibility and selection criteria}

Two authors extracted all experimental and clinical studies that met our search criteria. Additionally, the reference list of the articles included was investigated manually. No restriction was performed on the year and language of our search. After the search was completed, we removed the duplicates and screened the remaining articles. Articles that 
did not meet our inclusion criteria were removed from the list of references during the reading of the title, abstract and full texts. The outcomes of interest were cloth masks, filtration efficiency, penetration, pressure drop, and quality factor. Studies that refer to one or more of the above outcomes are included in our study. The inclusion criteria did not include any editorials, reviews and meta-analyses, reports and conference papers, and articles with insufficient data.

\section{Data extraction}

Data are summarized in the table (Table 1) based on a predefined checklist. The author's name, date, and place of the study, study type, sample size, identity and size of the particles, air flow rate or velocity, mask type, primary results, and risk of bias were extracted and summarized. All procedures of literature search, study selection, and data extraction were performed separately by two researchers. Any disagreement in the selection of articles has been resolved through discussion and consensus.

\section{Quality assessment}

The checklist evaluated all laboratory-based quasi-experimental studies (non-randomized experimental studies). For clinical trials, the checklist of randomized control trials (RCTs) from the Joanna Briggs Institute (JBI) was used (Tufanaru et al. 2017). The evaluated criteria were divided into nine areas for experimental studies and thirteen areas for clinical trials, categorized with "yes," "no," "unclear," or "not applicable." The checklists were analyzed for each study and classified by two authors as low, moderate, or high risk of bias. This final classification was assigned to the number of areas where "no" or "not applicable" were given as an answer. Thus, one or two domains were considered low risk in the experiment, three or four as moderate risk, and five or more as high risk of bias (Santos et al. 2020). In RCTs, one or two domains were included, while three or four were excluded, and five or more needed more information.

\section{Results}

\section{Study selection}

A total of 1163 records were primarily identified in the three electronic databases searched: PubMed, Web of Science, and two records from the reference list of other studies (Fig. 1). After the endnote manager removed 381 duplicates, 718 titles and abstracts were examined. Seventy records that satisfied the inclusion/exclusion criteria were retained for full-text assessment. Finally, forty-four articles have been selected and included in the qualitative synthesis of this systematic review. The summaries of the qualitative and quantitative data are shown in Table 1, respectively.

\section{Study characteristics}

The 44 final studies included in this systematic review consisted of three randomized control trials (RCTs) and 41 laboratory studies. The sample size, including different cloth mask models, was between 1 and 48. In three RCTs, the sample size was the number of people who participated in the trial, between 211 and 569. More than half of the studies $(n=21)$ researched cloth materials, and the other half investigated commercial cloth masks. In these experiments, sodium chloride $(\mathrm{NaCl})$ particles used more than all particles to examine different masks. Seventeen studies used $\mathrm{NaCl}$ particles in a size range of $0.009-10 \mu \mathrm{m}$, the flow rate was between $0.1-85 \mathrm{~L} \cdot \mathrm{min}^{-1}$, and the velocity was in the range of 5.3-1650 cm.s ${ }^{-1}$ (Bowen 2010; Clapp et al. 2020; Drewnick et al. 2021; Hao et al. 2020; Joshi et al. 2020; Konda et al. 2020a; Liu et al. 2019; Long et al. 2020; Mueller et al. 2020; O'Kelly et al. 2020; Park and Jayaraman 2020; Pei et al. 2020; Rengasamy et al. 2010; Varallyay et al. 2020; Wang et al. 2020; Zangmeister et al. 2020; Zhao et al. 2020; Guha et al. 2021). In addition to sodium chloride, three studies used another particle. One of them used $0.101 \mu \mathrm{m}$ polystyrene latex (PSL) particles with a velocity of $10 \mathrm{~cm} . \mathrm{s}^{-1}$ (Lu et al. 2020). In the other one, paraffin oil aerosols were utilized with the $0.225 \mu \mathrm{m}$ count median diameter (Jung et al. 2013). The third one used KCL + sodium fluorescein with $0-7 \mu \mathrm{m}$ particle size range and $28.3 \mathrm{~L} / \mathrm{min}$ flow rate (Lindsley et al. 2021). Nine studies used different particles that ranged in the size of $0.001-10 \mu \mathrm{m}$; the flow rate was between 0.9-300 L. $\mathrm{min}^{-1}$ (Aydin et al. 2020; Chen et al. 2013; Cherrie et al. 2018; Lustig et al. 2020; Maher et al. 2020; Neupane et al. 2019; Pacitto et al. 2019; Shakya et al. 2017; Xiao et al. 2020). One study reported a velocity of $44.4 \mathrm{~m} . \mathrm{s}^{-1}$ (Xiao et al. 2020), and another study reported the velocity of $17.1 \mathrm{~m} . \mathrm{s}^{-1}$ (Aydin et al. 2020). Five studies used virus and bacteria particles to measure the efficiency of masks (Davies et al. 2013; Ma et al. 2020; Rodriguez-Palacios et al. 2020; Ueki et al. 2020; Whiley et al. 2020). These particle sizes ranged from $0.023-1000 \mu \mathrm{m}$. In one study, the flow rate was 30 L. $\mathrm{min}^{-1}$ (Davies et al. 2013). Four other studies did not mention the flow rate or velocity (Ma et al. 2020; RodriguezPalacios et al. 2020; Ueki et al. 2020; Whiley et al. 2020). Three studies designed the experiment with respiratory particles produced by breathing, coughing, and talking that ranged from 0.01 to $20 \mu \mathrm{m}$ and the flow rate was in the range of 5-80 L.min ${ }^{-1}$ (Asadi et al. 2020; Li et al. 2020b; van der Sande et al. 2008). Except for three studies that used human subjects (Asadi et al. 2020; Clapp et al. 2020; van der Sande 
1648

Environmental Science and Pollution Research (2022) 29:1645-1676

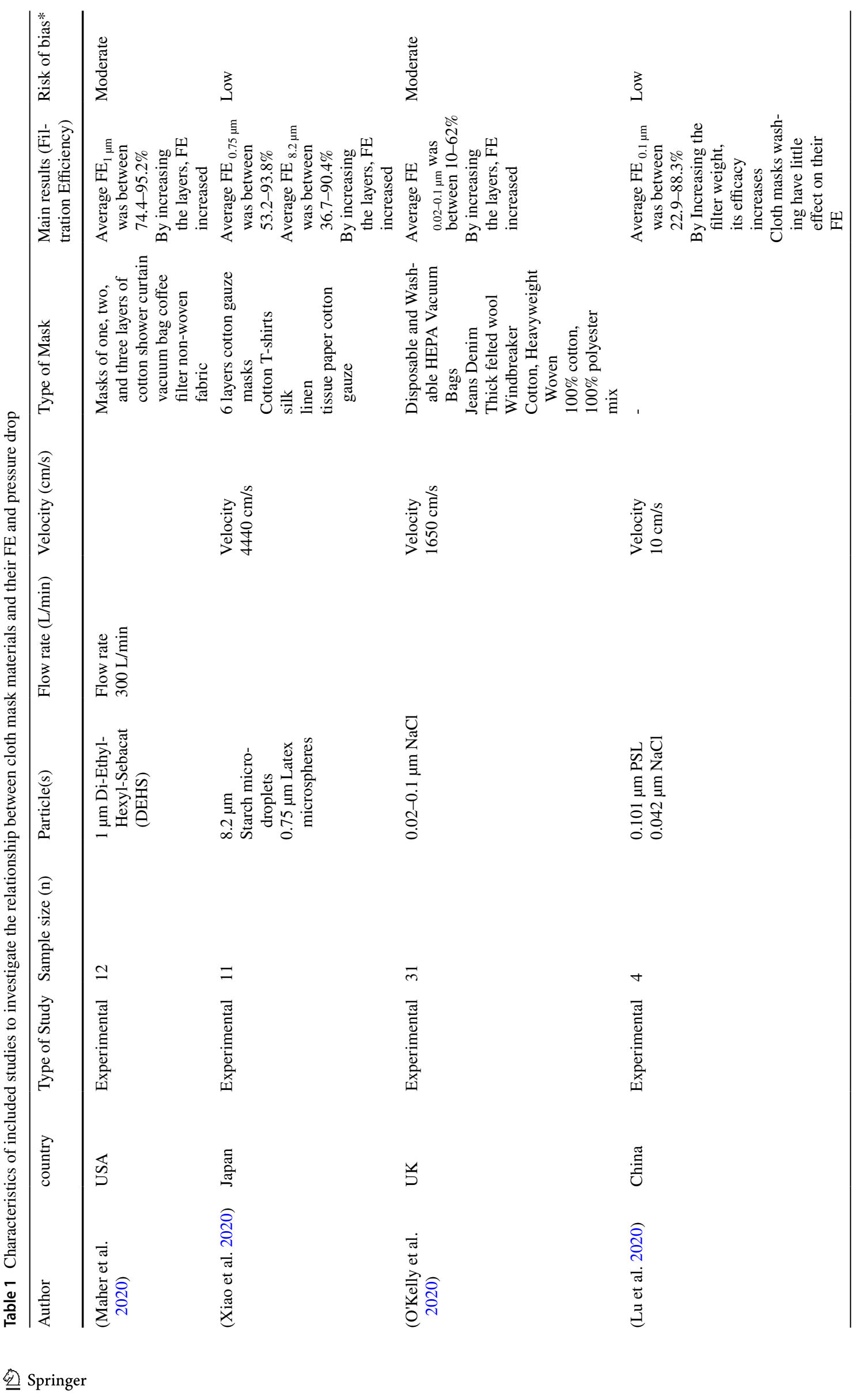




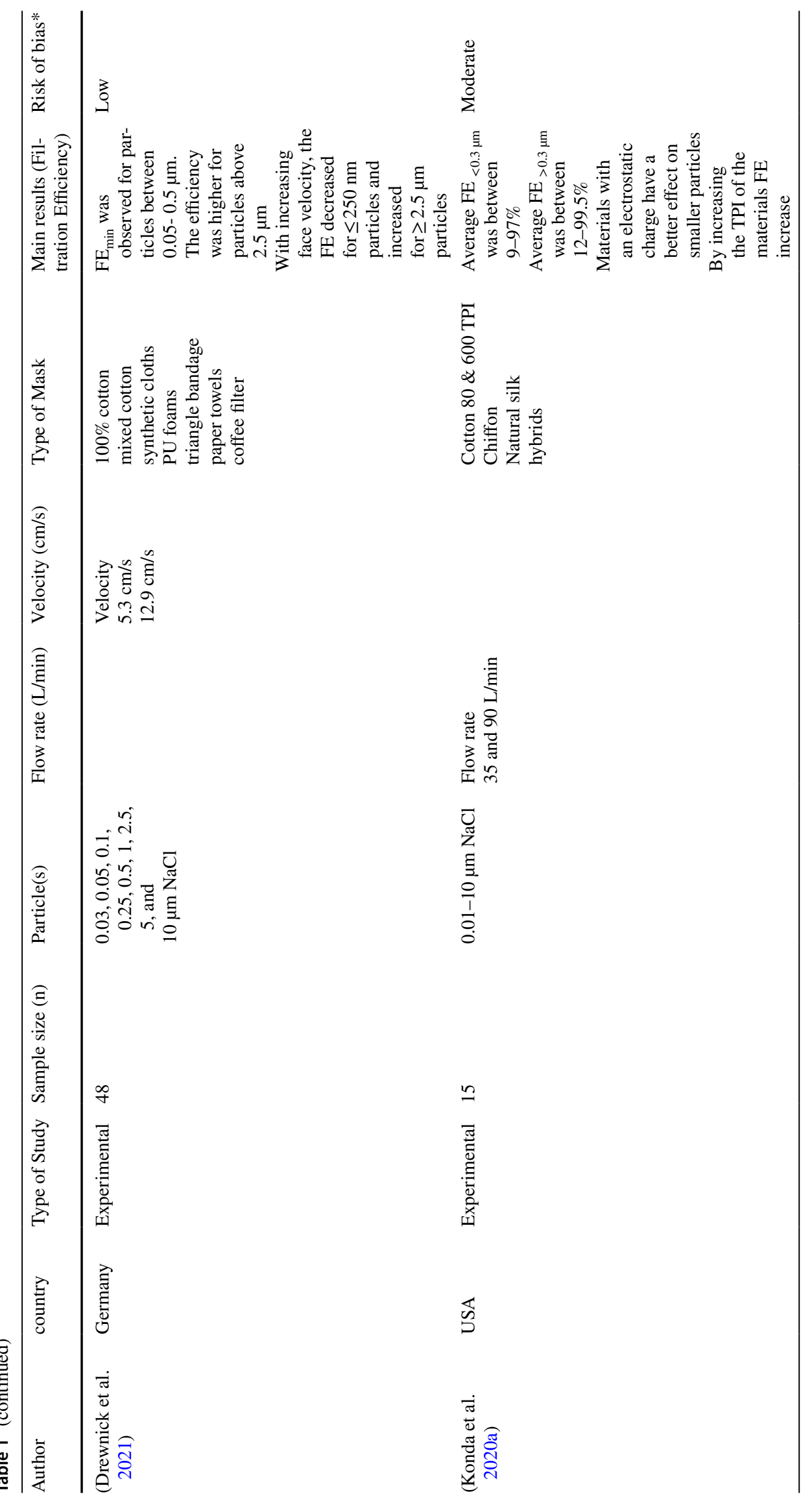




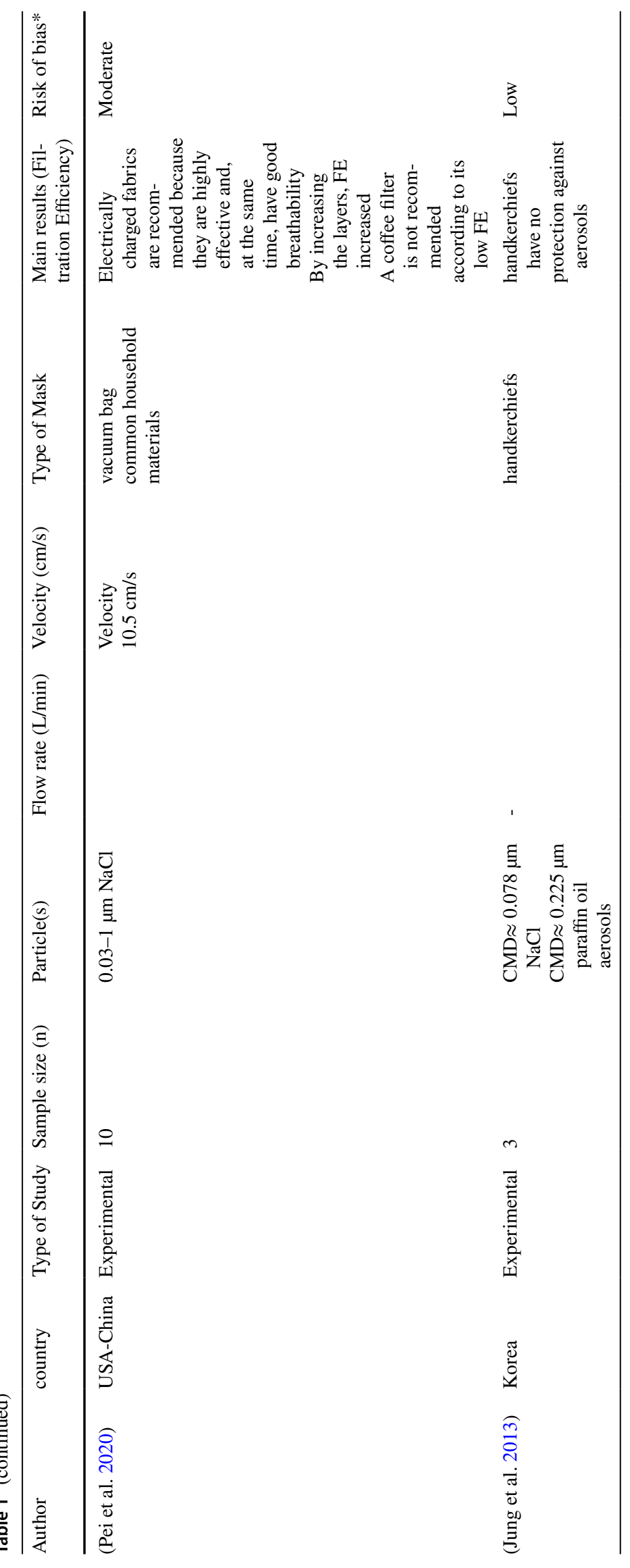




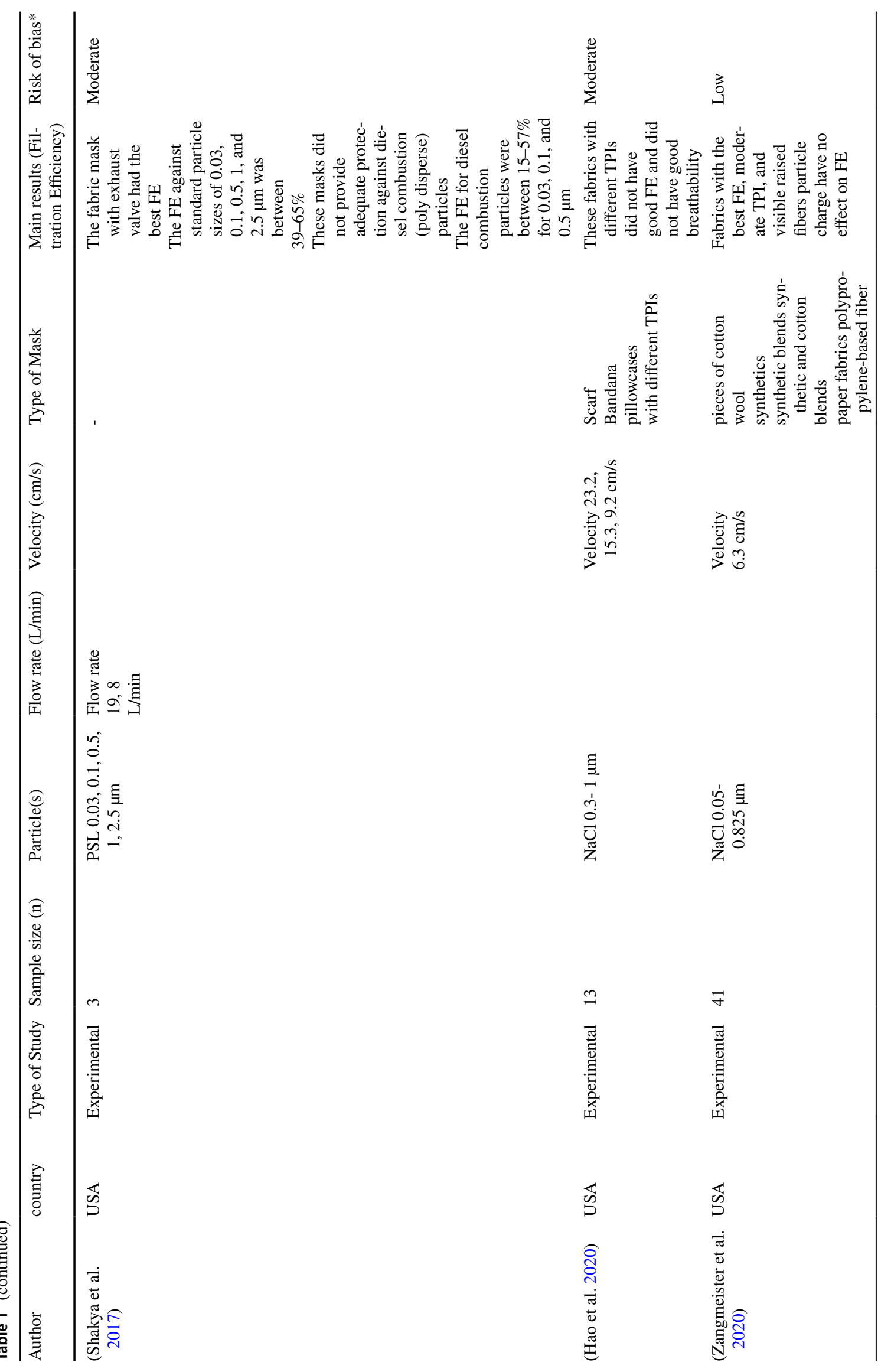




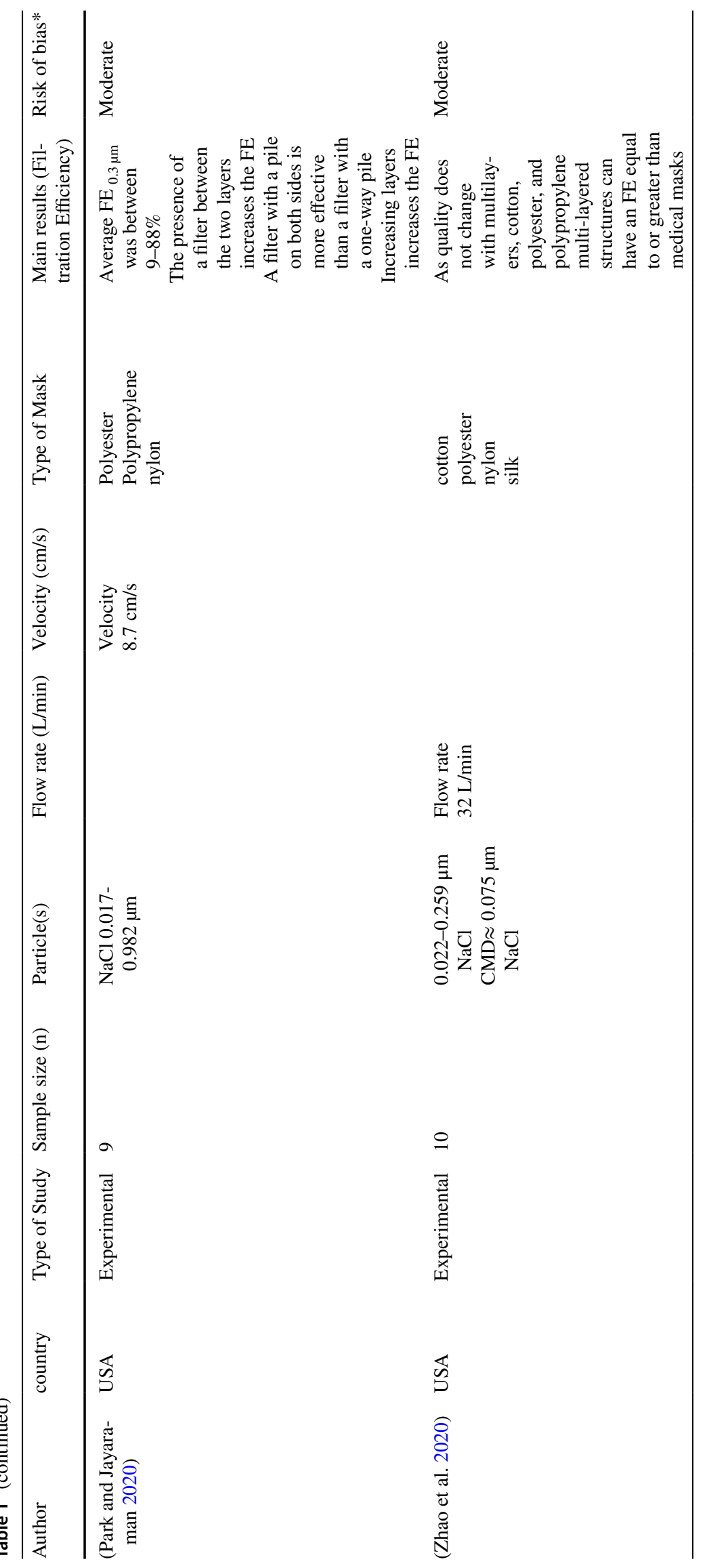




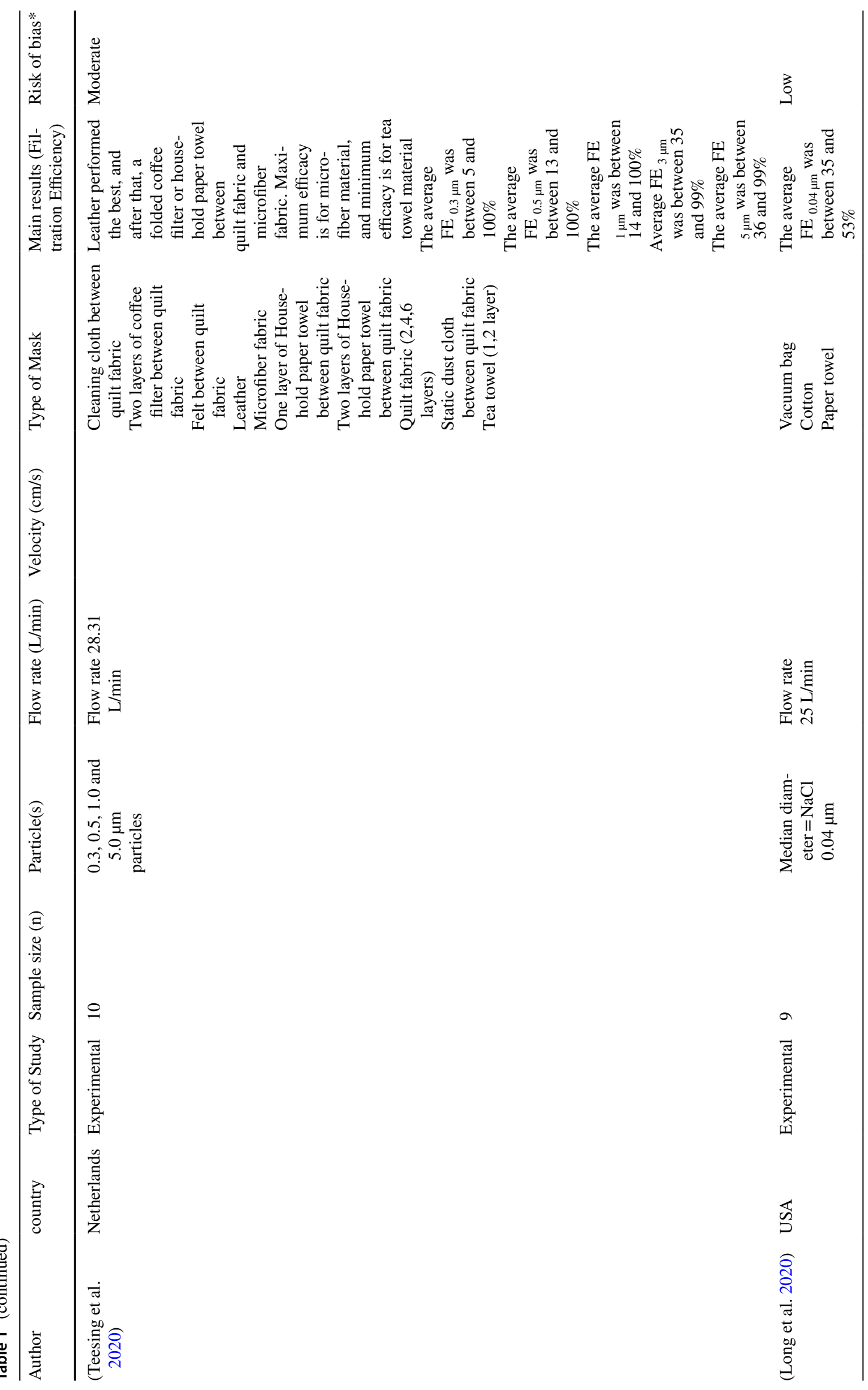




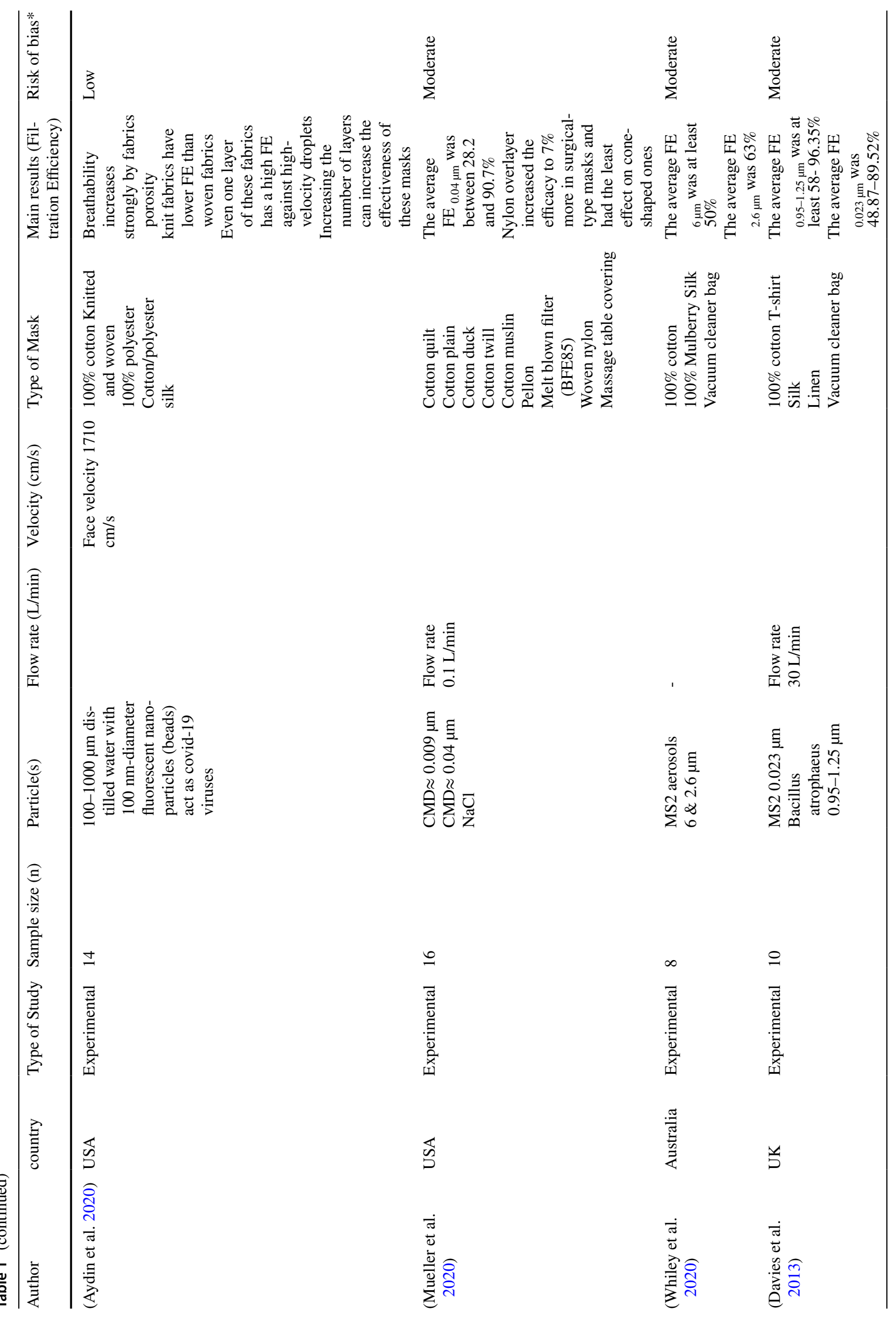




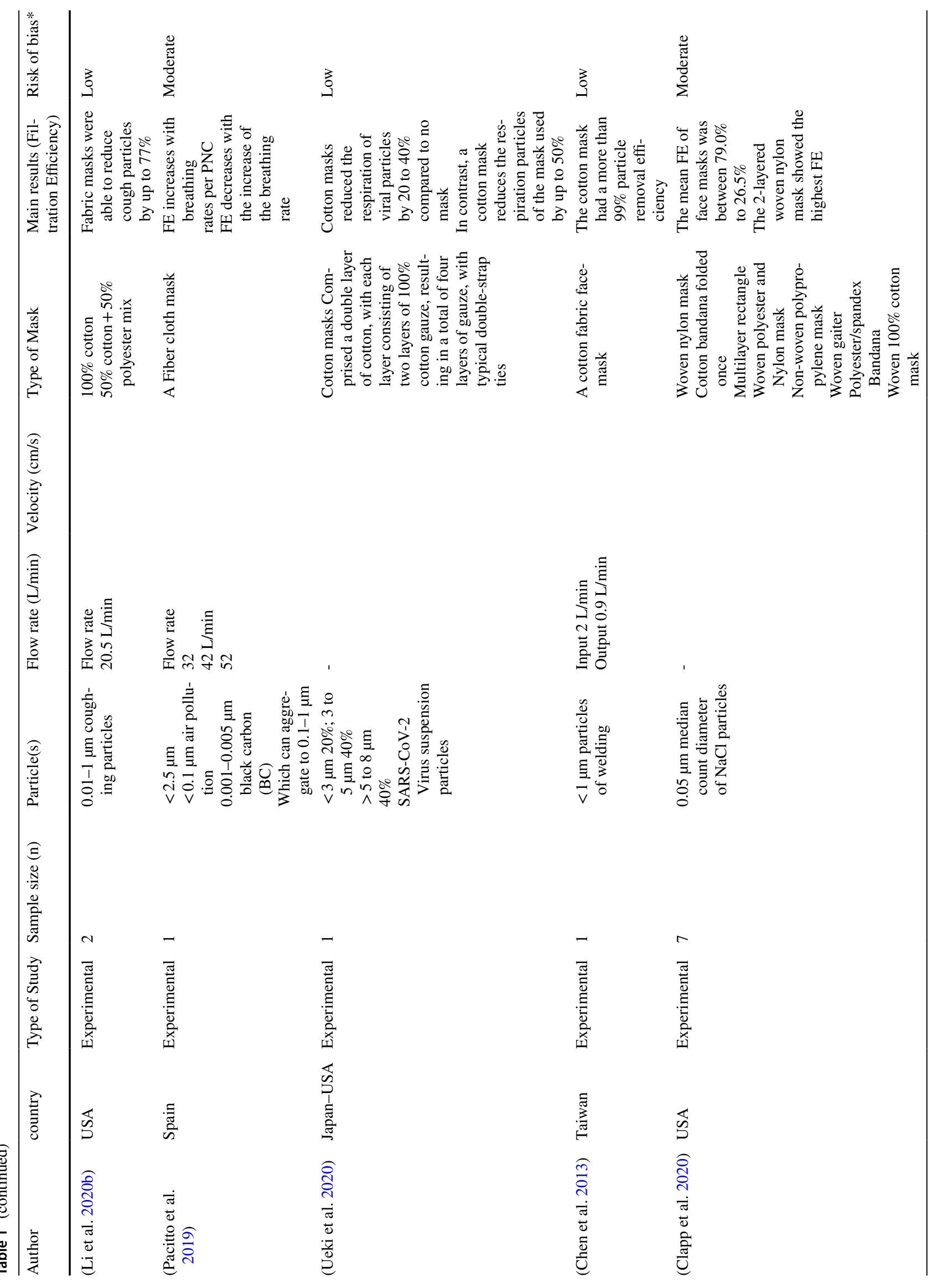




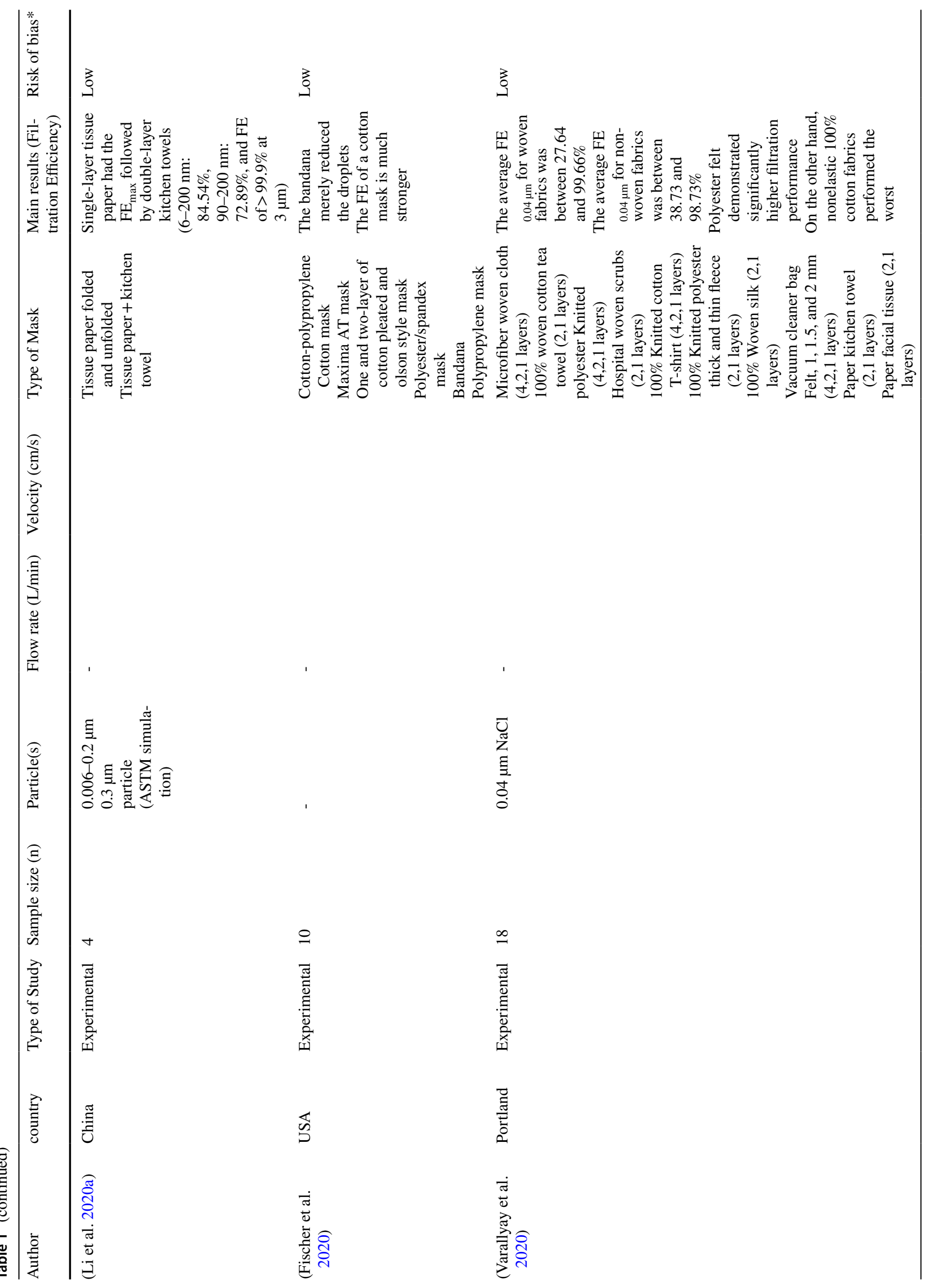




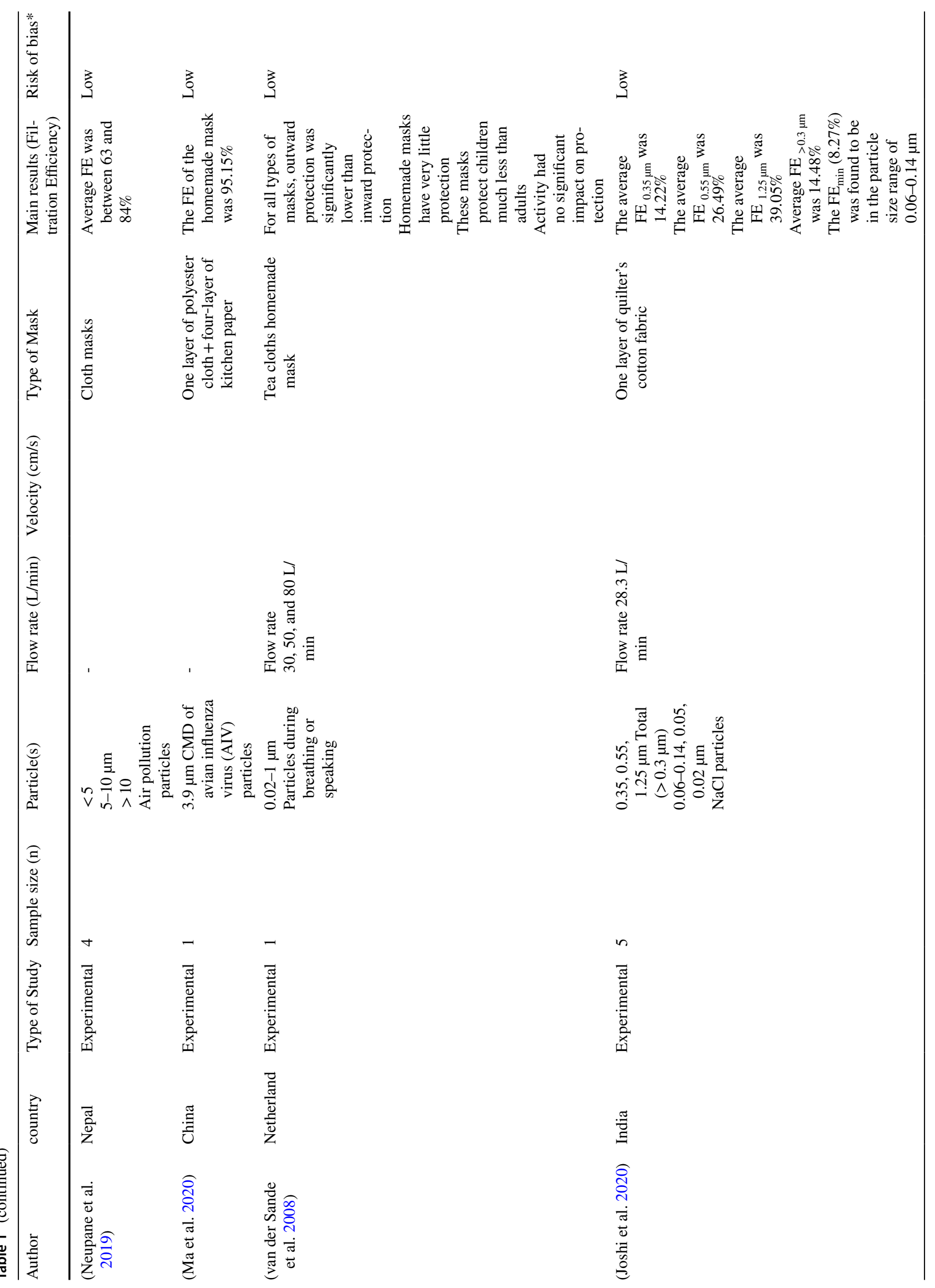




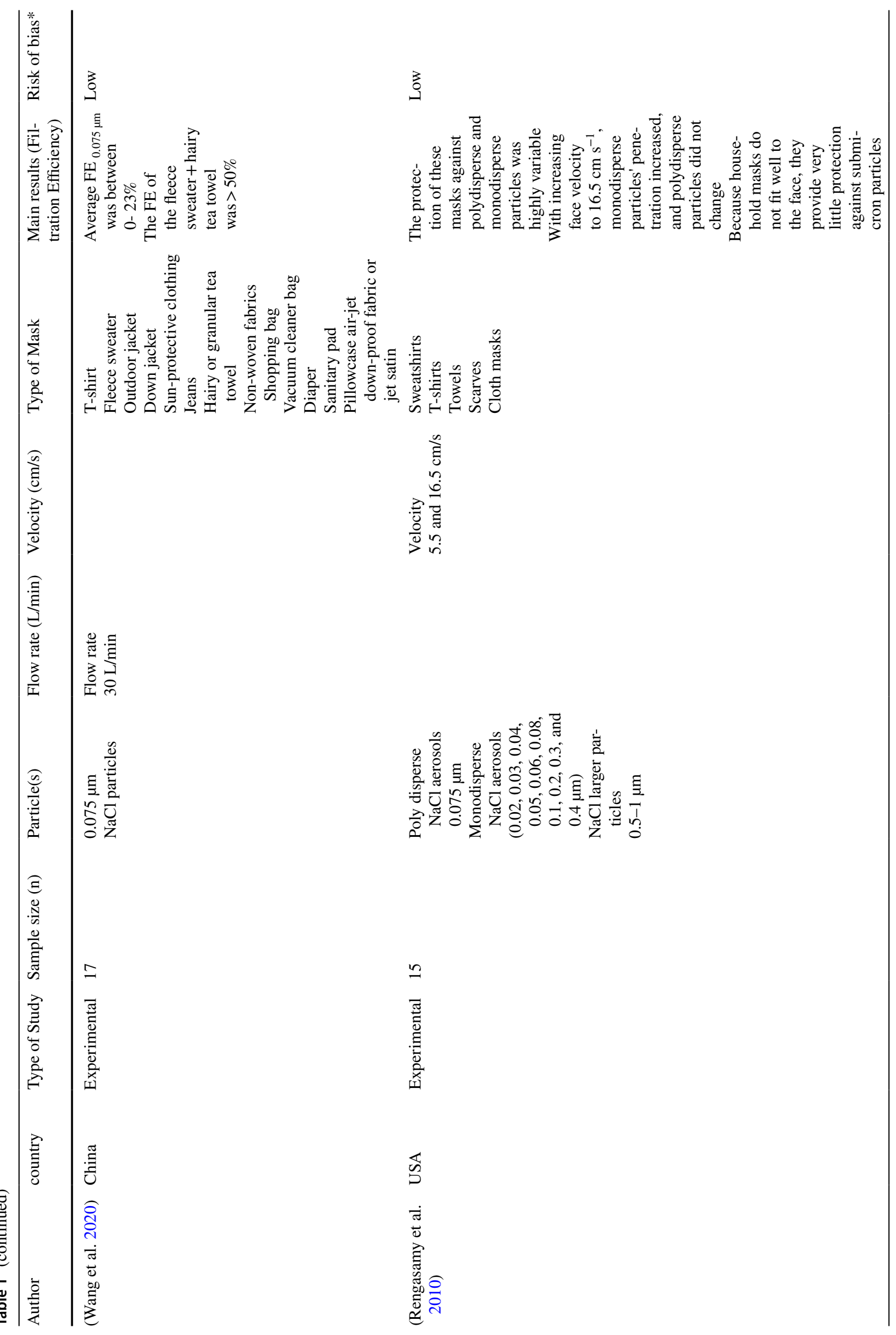




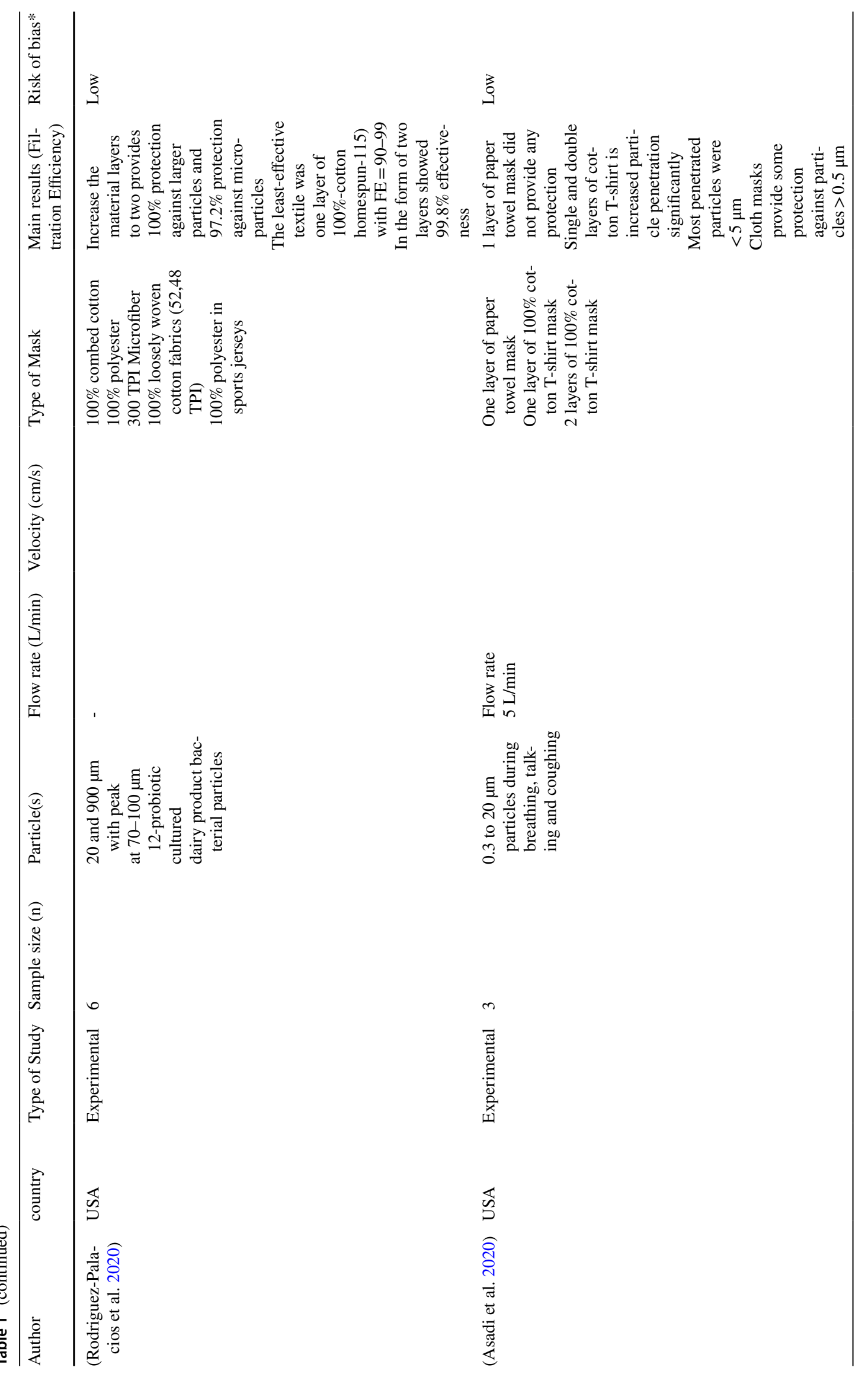




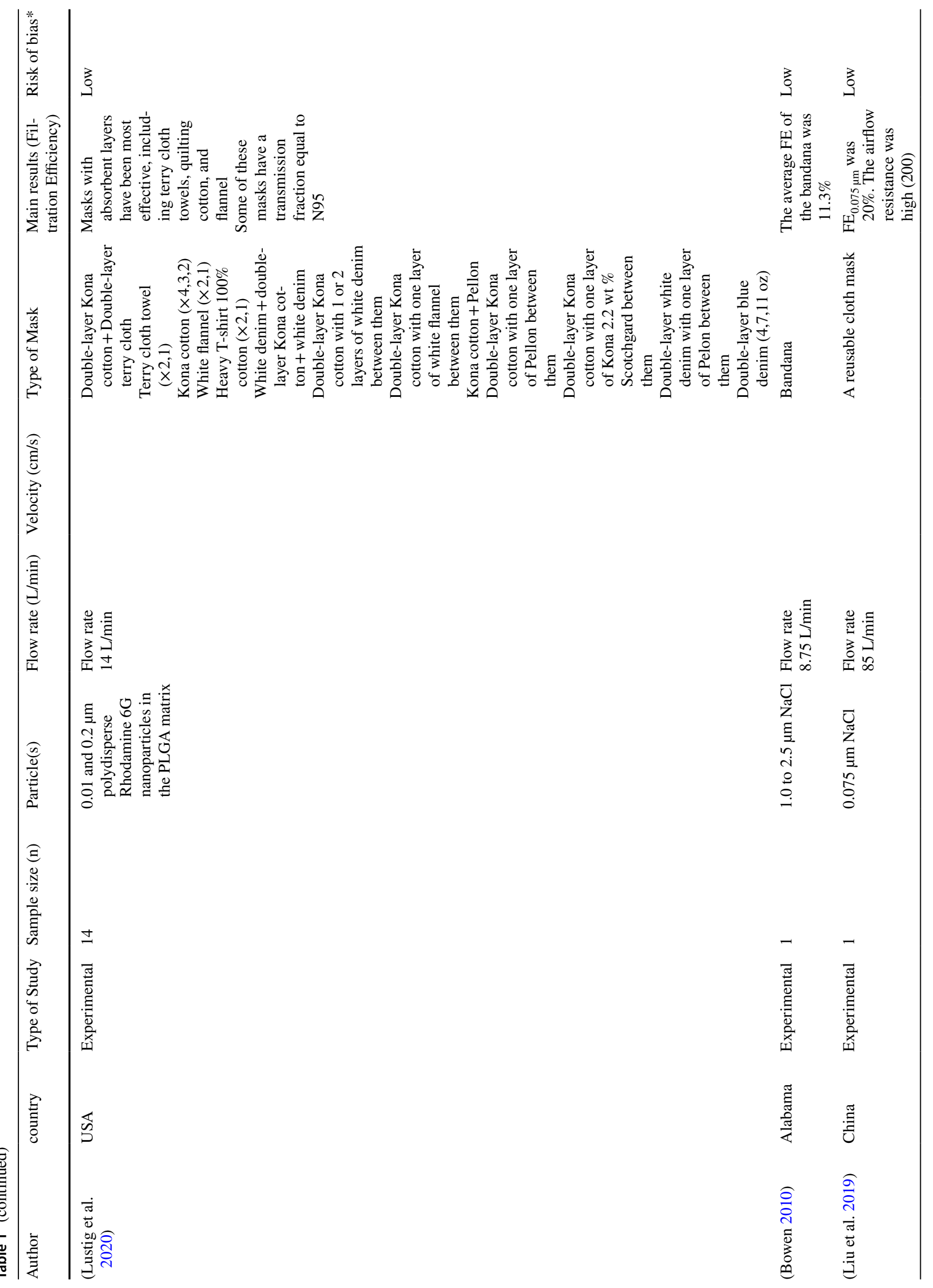




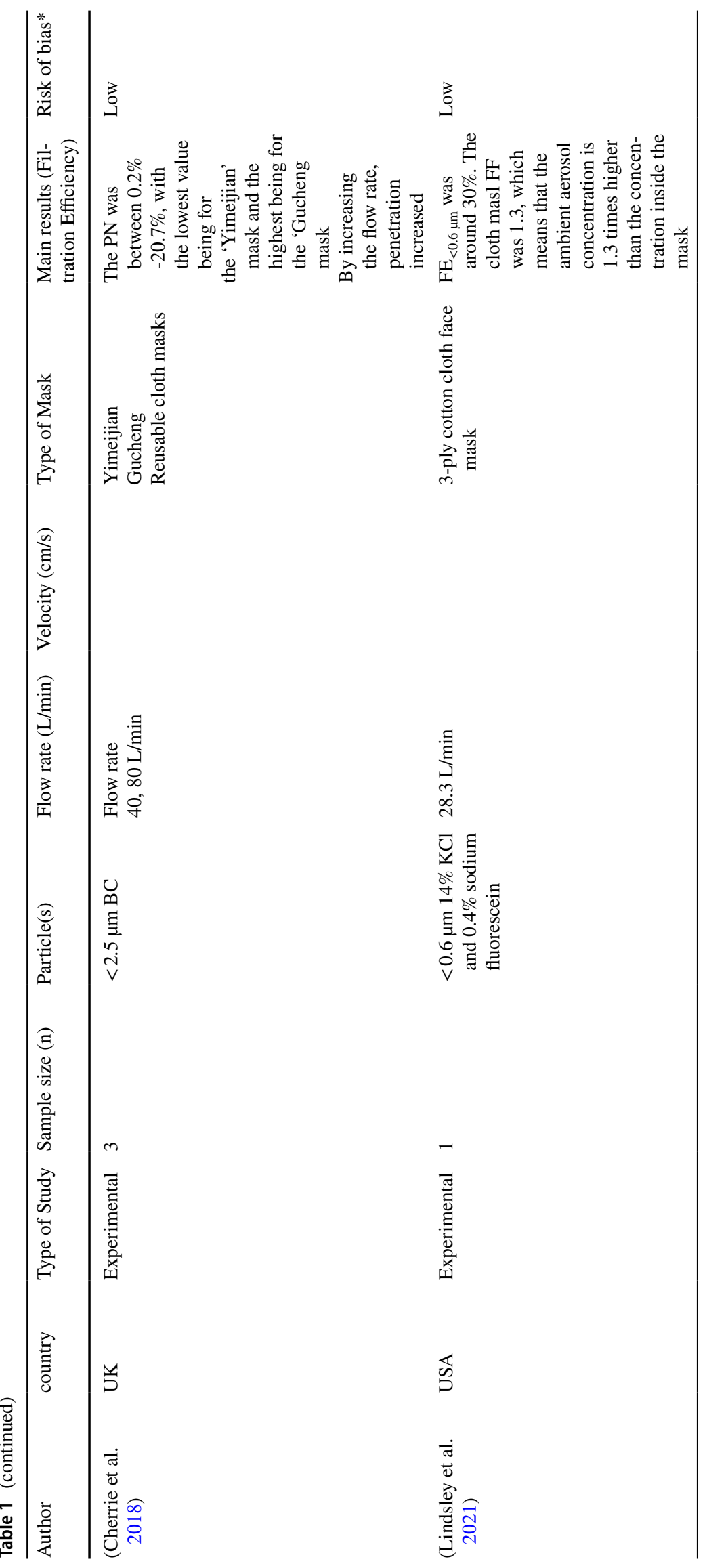




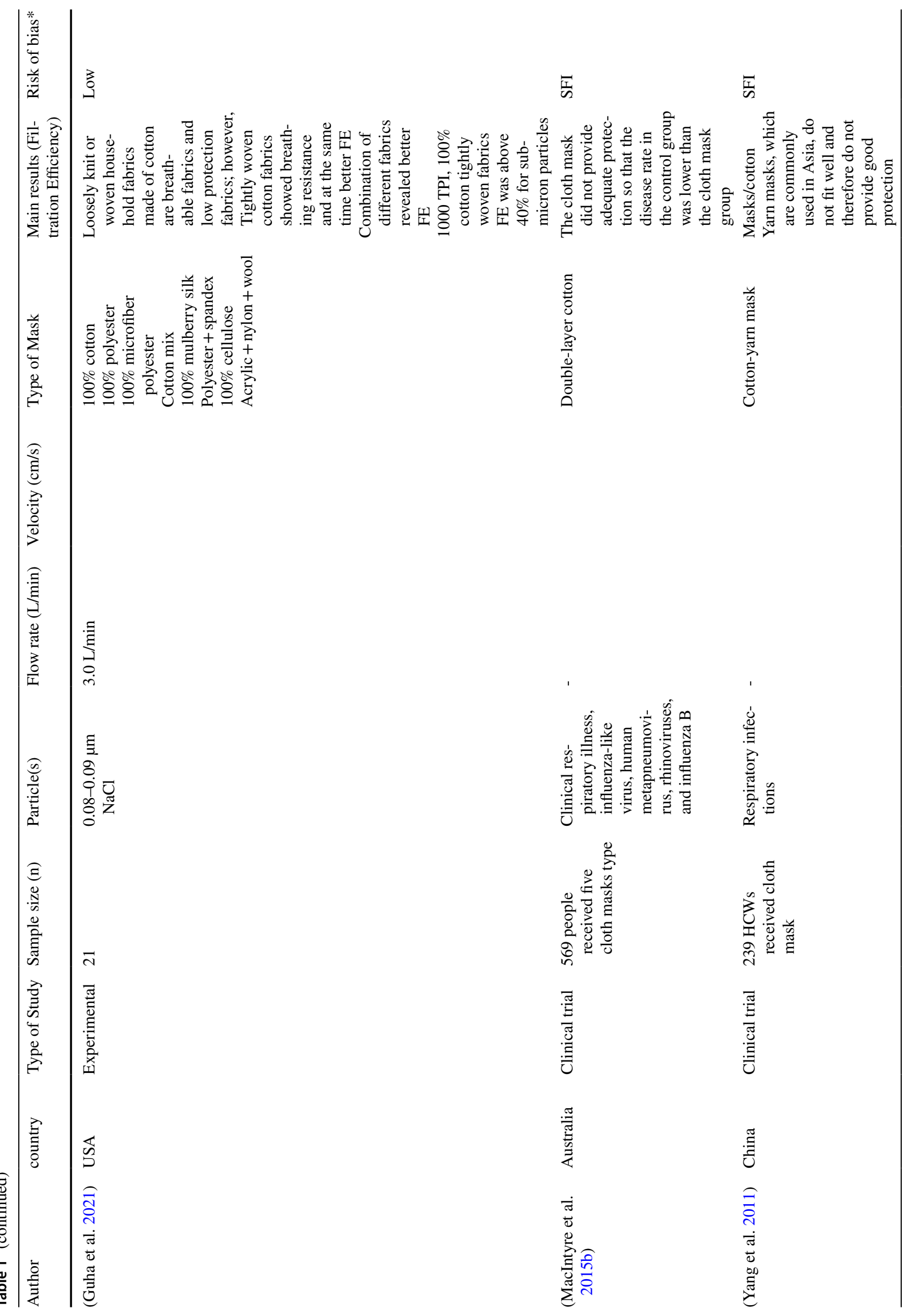


et al. 2008), all other thirty-seven experimental studies used manikin-based models. Clinical studies measured the mask's efficiency by health care workers who were infected by different respiratory viruses (Ho et al. 2020; MacIntyre et al. 2015b; Yang et al. 2011).

\section{Systematic review}

\section{Filtration efficacy (FE)}

By measuring the pre and post mask viral aerosols concentration, the FE will be calculated by this formula:Efficiency $(\%)=\left(1-\frac{B}{A}\right) \times 100 \%$. A: refers to the concentration of viral aerosol challenging the mask, and $\mathrm{B}$ is the concentration of viral aerosol after mask filtration (Wen et al. 2010). The FE in forty-one experimental studies was reported in the range of $0-100 \%$. Four studies found $\mathrm{FE}$ increases by increasing the layer of clothes (Maher et al. 2020; O'Kelly et al. 2020; Xiao et al. 2020; Guha et al., 2021). One study added a nylon layer to different cloth masks and obtained the FE of surgical type masks increased but it had no effect on cone-shaped masks (Mueller et al. 2020). Guha et al. study also revealed that a combination of woven and loosely knitted fabrics can increase the FE against sub-micron particles (Guha et al. 2021). Two different studies concluded that by increasing the weight of filter material (Lu et al. 2020) and the thread per inch (TPI) (Konda et al. 2020a), FE increased. Additionally, FE depends on different parameters, including particle size, and flow rate through the filter material (Cherrie et al. 2018). Studies showed that by increasing the flow rate and/or face velocity, FE decreases (Shakya et al. 2017; O'Kelly et al. 2020). However, Lu et al. asserted that velocity increasing (from 4 to $16 \mathrm{~cm} \mathrm{~s}^{-1}$ ) has no effect on FE (Lu et al. 2020). We have to keep this point in mind that in different studies flow rate is not constant. To be sure about the comparisons, new studies with the same situation should be done. Twelve studies calculated the filtration efficiency of different materials in the particle size range of the Covid-19, which (60-100 nm) was between 0-97\% (Joshi et al. 2020; Konda et al. 2020a; Li et al. 2020a, b; O'Kelly et al. 2020; Pei et al. 2020; Shakya et al. 2017; Wang et al. 2020; Zangmeister et al. 2020; Zhao et al. 2020; Lindsley et al. 2021; Guha et al., 2021) (Table 2). According to Konda et al. study, the most effective cloth mask was a hybrid of cotton/chiffon (). They compared different fabric materials with a separate TPI and a different number of layers (Konda et al. 2020a). Then they selected some material that had a better performance to combine. Finally, between different tested materials, cotton quilt (120 TPI) $(\mathrm{FE}=96 \%)$, and among different hybrid masks, hybrid of cotton/chiffon ( $\mathrm{FE}=97 \%$ ), hybrid of cotton/silk (no gap) $(\mathrm{FE}=94 \%)$, hybrid of cotton/flannel $(\mathrm{FE}=95 \%)$ had the best filtration efficiency. Albeit, we have 
Fig. 1 PRISMA Flowchart of the literature search and strategy for the selection of relevant studies

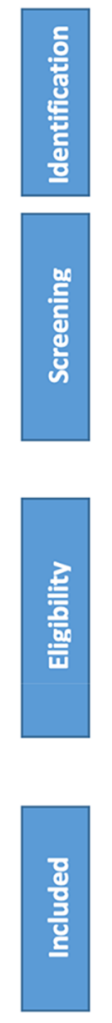

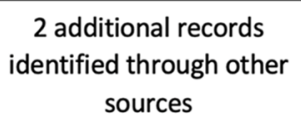

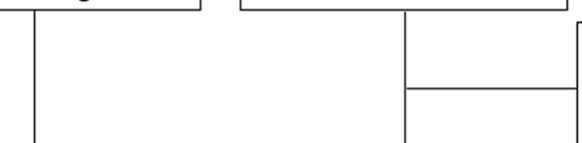

381 duplicate studies excluded during initial screen

\section{Records screened}

70 Full-text articles assessed for eligibility

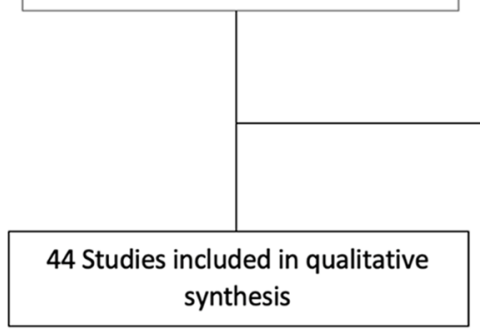

648 studies were excluded after reading the title and abstracts record: 582 Were irrelevant, 2 were duplicates, 64 were review article, letters, case report, animal studies.

30 studies that did not report outcomes of Interest were excluded after reading Full-text of articles to mention some defects of this study. Carr et al. published a letter and criticized this study methods. According to Carr et al. study the pressure drop values were significantly lower compared to similar articles (Carr et al. 2020). Furthermore, FE of N95 reported 45-70\%, which was controversial. In response, Konda et al. corrected that the N95 and cloth masks capturing efficacy measured in a significantly lower pressure drop $(2.5-13 \mathrm{~Pa}$ ) than similar studies (Konda et al. 2020b). The Pillowcase $80 \mathrm{~s} \times 60 \mathrm{~s}$ Jet satin had no efficacy (Wang et al. 2020). Zangmeister et al. examined different cloth materials with a different number of layers. The bestperforming materials were $100 \%$ cotton fabrics, including down-proof ticking, woven hand towel, light-weight flannel, and a 4-layer 100\% cotton light-weight flannel (poplin) with a FE of 48\% (Zangmeister et al. 2020). By evaluating different cloth materials, Zhao et al. found that cellulose copy paper (bonded) had the best $99.85 \%$ FE (Zhao et al. 2020). Pei et al. evaluated five layers of different materials; a 5-layer shop towel with $69 \%$ FE had better performance (Pei et al. 2020). In a study by Li et al. several cloth materials have been examined and then reported a mixture of tissue paper and kitchen towels with $71.5 \% \mathrm{FE}$ that performed the best (Li et al. 2020a). The Joshi et al. study tested just a singlelayered quilter's cotton fabric (TPI $=85-100$ ) that had an inadequate FE against particles in size range of $60-140 \mathrm{~nm}$ $(\mathrm{FE}=8.27 \%)$ (Joshi et al. 2020). Wang et al. compared different materials; the results showed that all of the materials had low FE, but hairy tea towel $80 \%$ polyester $20 \%$ nylon with 23\% FE was the best (Wang et al. 2020). O'Kelly et al. reported that disposable HEPA Vacuum Bags filtered more than $60 \%$ of $20-1000 \mathrm{~nm}$ particles (O'Kelly et al. 2020). In another study by Li et al., 2-ply $100 \%$ cotton masks showed $77 \%$ FE for 10-1000 nm particles (Li et al. 2020b). Shakya et al. compared 3 different cloth masks. One of them had an exhalation valve and, the others did not have it. In this study, the cloth mask with exhalation valve showed $\approx 90 \%$ FE for $100 \mathrm{~nm}$ particles (Shakya et al. 2017). Lindsley et al. study, revealed the $\approx 28 \%$ FE of 3 -ply cotton face masks for particles ranged between 0-600 $\mathrm{nm}$ (Lindsley et al. 2021). And the Guha et al. reported the One Thousand TPI Bedsheet-1 (1000 TCBS1) with $48.9 \% \mathrm{FE}$ was the best performing onelayered fabric which showed $\approx 5 \%$ FE increase after adding up another layer (Guha et al. 2021).

Moreover, Table 3 shows different surrogates tested which $\mathrm{NaCl}$ was the most used one. Among those tested $\mathrm{NaCl}$ particles, copy paper (bonded) indicated the highest FE. All these results can be seen in Fig. 2.

Two clinical trials reported that cloth masks' efficacy was low, and the rate of respiratory infections in the cloth mask wearers was high (MacIntyre et al. 2015b; Yang et al. 2011). The previous clinical study found no difference between the cotton mask and a medical mask (Ho et al. 2020). 
Table 2 Summary of studies that evaluated cloth materials in the Covid-19 particle size range

\begin{tabular}{|c|c|c|c|c|}
\hline Mask type & $\begin{array}{l}\text { Filter } \\
\text { efficiency } \\
(\%)\end{array}$ & $\begin{array}{l}\Delta \mathrm{P} \\
(\mathrm{Pa})\end{array}$ & $\mathrm{QF}(\mathrm{kPa}-1)$ & Particle size \\
\hline $100 \%$ cotton hand towel (block), 2-layers & 32 & 61.8 & 6.25 & \multirow{7}{*}{$\begin{array}{l}50-825 \mathrm{~nm} \\
\mathrm{NaCl} \text { method (Zangmeister et al. 2020) }\end{array}$} \\
\hline $100 \%$ cotton light weight flannel (poplin), 2-layers & 24.3 & 106 & 2.62 & \\
\hline $100 \%$ cotton light weight flannel (poplin), 4-layers & 48 & 216 & $<2.62$ & \\
\hline $100 \%$ cotton pillowcase (satin), 2-layers & 20.3 & 128.5 & 1.77 & \\
\hline Polyester apparel fabric (Poplin), 2-layers & 21.4 & 104 & 2.32 & \\
\hline Polyester apparel fabric (soft spun), 2-layers & 20.2 & 177.6 & 1.27 & \\
\hline Coffee filter & 34.4 & - & - & \\
\hline polypropylene 4 (PP-4) & 6.1 & 1.6 & 16.9 & \multirow[t]{10}{*}{ 22-259 nm NaCl NIOSH method (Zhao et al. 2020) } \\
\hline Cotton pillow cover (woven) & 5.04 & 4.5 & 5.4 & \\
\hline Cotton T-shirt (knit) & 21.62 & 14.5 & 7.4 & \\
\hline Cotton sweater (knit) & 25.88 & 17.0 & 7.6 & \\
\hline Polyester toddler wrap (knit) & 17.50 & 12.3 & 6.8 & \\
\hline Silk napkin (woven) & 4.77 & 7.3 & 2.8 & \\
\hline Nylon exercise pants (woven) & 23.33 & 244.0 & 0.4 & \\
\hline Paper towel (bonded) & 10.41 & 11.0 & 4.3 & \\
\hline Tissue paper (bonded) & 20.2 & 19.0 & 5.1 & \\
\hline Copy paper (bonded) & 99.85 & 1883.6 & 1.5 & \\
\hline Coffee filter, 2 layers & 14 & 153.4 & - & \multirow{5}{*}{$\begin{array}{l}100 \mathrm{~nm} \\
\mathrm{NaCl} \text { NIOSH method (Pei et al. 2020) }\end{array}$} \\
\hline Kitchen towel, 5 layers & 40 & 158.9 & & \\
\hline Bed sheet, 5 layers & 54 & 433.9 & & \\
\hline T-shirt, 5 layers & 64 & 231.1 & & \\
\hline Shop towel, 5 layers & 69 & 185.8 & & \\
\hline 4-ply tissue paper & 30.4 & - & - & \multirow{3}{*}{$\begin{array}{l}100 \mathrm{~nm} \\
\text { ASTM method (Li et al. 2020a) }\end{array}$} \\
\hline 4-ply tissue paper folded once & 41.2 & & & \\
\hline Tissue paper + kitchen towel & 71.5 & & & \\
\hline Disposable HEPA Vacuum Bags & 60.86 & 2 & - & \multirow{20}{*}{$\begin{array}{l}20-1000 \mathrm{~nm} \\
\mathrm{NaCl} \text { method (O'Kelly et al. 2020) }\end{array}$} \\
\hline Windbreaker $100 \%$ Polyester & 47.12 & 3 & & \\
\hline Jeans Denim 100\% Cotton & 45.94 & 3 & & \\
\hline Washable Vacuum Bag HEPA & 43.64 & 2 & & \\
\hline Thick felted wool $100 \%$ & 35.87 & 0 & & \\
\hline Cotton, Heavyweight Woven $100 \%$ & 35.77 & 2 & & \\
\hline Folded Sock Cotton & 35.36 & 2 & & \\
\hline Quilting Cotton $100 \%$ & 34.54 & 1 & & \\
\hline Two-Sided Minky Fabric & 34.17 & 1 & & \\
\hline Shirting Cotton $100 \%$ & 33.59 & 1 & & \\
\hline Cotton, Lightweight Woven $100 \%$ & 30.2 & 0 & & \\
\hline Cotton Quilt Batting 100\% & 29.81 & 0 & & \\
\hline Cotton Flannel 100\% & 28.5 & 1 & & \\
\hline Craft Felt Miss crafts Rayon, Acrylic, Polyester & 27.72 & 0 & & \\
\hline $100 \%$ Nylon Woven & 27.61 & 3 & & \\
\hline T-shirt, Heavyweight $100 \%$ Cotton & 25.21 & 1 & & \\
\hline Cotton Jersey Knit 100\% Cotton & 24.56 & 0 & & \\
\hline Lycra $82 \%$ Nylon, $18 \%$ Spandex & 21.6 & 0 & & \\
\hline Fusible Interfacing HTC & 15 & 0 & & \\
\hline T-Shirt ( $50 \%$ Polyester $+50 \%$ Cotton) & 10.5 & 0 & & \\
\hline Quilter's cotton fabric (TPI $=85-100), 1$ layer & 8.27 & - & - & $\begin{array}{l}60-140 \mathrm{~nm} \\
\mathrm{NaCl} \text { method (Joshi et al. 2020) }\end{array}$ \\
\hline
\end{tabular}


Table 2 (continued)

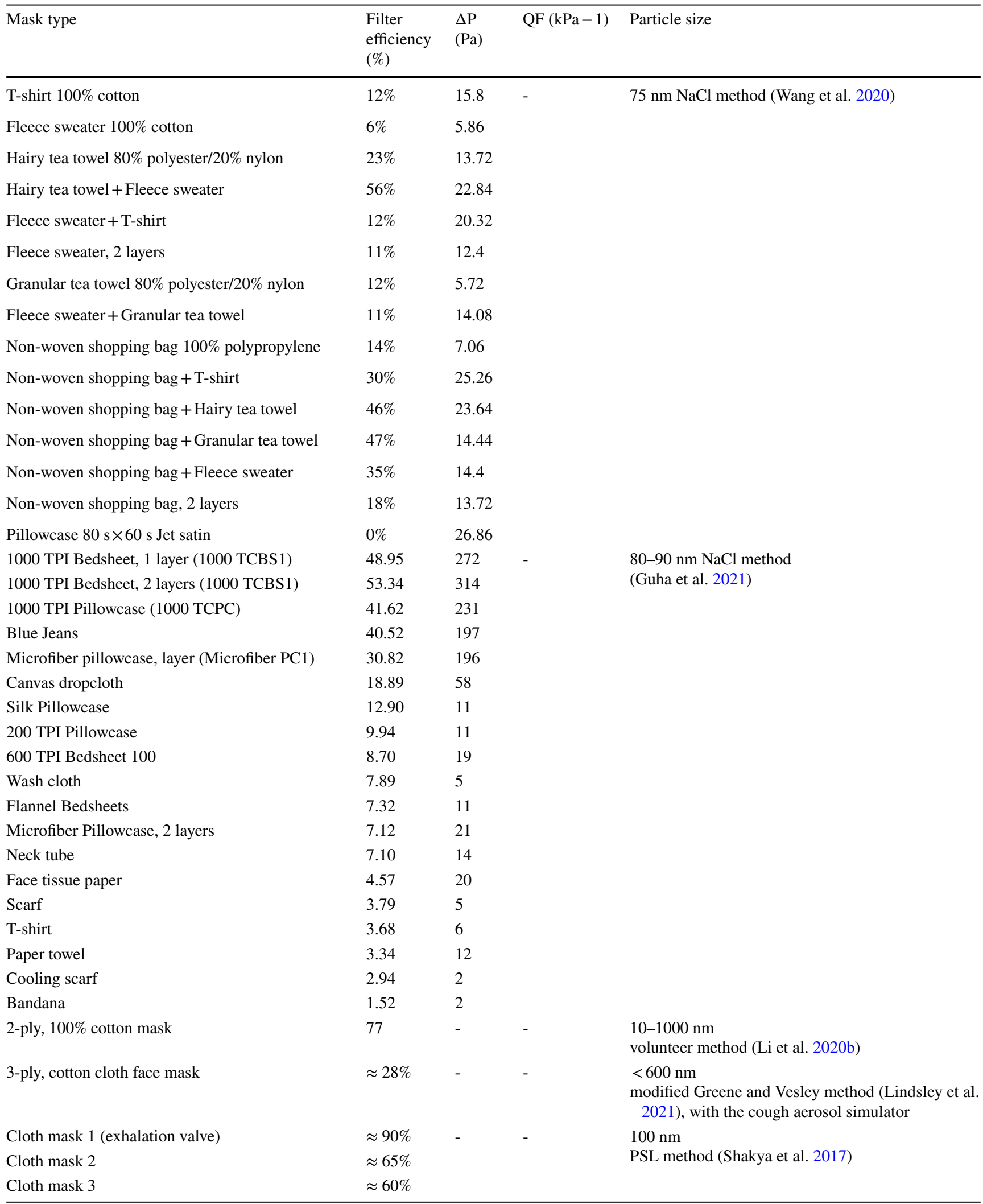


Table 3 Comparison of different study conditions

\begin{tabular}{|c|c|c|c|c|c|c|}
\hline Mask type & $\begin{array}{l}\text { Filter } \\
\text { efficiency } \\
(\%)\end{array}$ & Flow rate & Testing surrogates used & Particle size & Type of aerosols/droplets & $\begin{array}{l}\text { Electrostatic } \\
\text { charge of the } \\
\text { particles }\end{array}$ \\
\hline $\begin{array}{l}100 \% \text { cotton hand towel } \\
\text { (block), 2-layers }\end{array}$ & 32 & \multirow[t]{7}{*}{$2.2 \mathrm{~L} / \mathrm{min}$} & \multirow[t]{7}{*}{$\mathrm{NaCl}$} & \multirow[t]{7}{*}{$50-825 \mathrm{~nm}$} & \multirow[t]{7}{*}{$\begin{array}{l}\text { Solid } \\
\text { (Zangmeister et al. 2020) }\end{array}$} & \multirow[t]{7}{*}{ No } \\
\hline $\begin{array}{l}100 \% \text { cotton light weight } \\
\text { flannel (poplin), 2-layers }\end{array}$ & 24.3 & & & & & \\
\hline $\begin{array}{l}\text { 100\% cotton light weight } \\
\text { flannel (poplin), 4-layers }\end{array}$ & 48 & & & & & \\
\hline $\begin{array}{l}\text { 100\% cotton pillowcase } \\
\text { (satin), 2-layers }\end{array}$ & 20.3 & & & & & \\
\hline $\begin{array}{l}\text { Polyester apparel fabric } \\
\text { (Poplin), 2-layers }\end{array}$ & 21.4 & & & & & \\
\hline $\begin{array}{l}\text { Polyester apparel fabric } \\
\text { (soft spun), 2-layers }\end{array}$ & 20.2 & & & & & \\
\hline Coffee filter & 34.4 & & & & & \\
\hline polypropylene 4 (PP-4) & 6.1 & \multirow[t]{10}{*}{$32 \mathrm{~L} / \mathrm{min}$} & \multirow[t]{10}{*}{$\mathrm{NaCl}$ NIOSH method } & \multirow[t]{10}{*}{$22-259 \mathrm{~nm}$} & \multirow{10}{*}{$\begin{array}{l}\text { Solid } \\
\text { (Zhao et al. 2020) }\end{array}$} & \multirow[t]{10}{*}{ No } \\
\hline $\begin{array}{l}\text { Cotton pillow cover } \\
\text { (woven) }\end{array}$ & 5.04 & & & & & \\
\hline Cotton T-shirt (knit) & 21.62 & & & & & \\
\hline Cotton sweater (knit) & 25.88 & & & & & \\
\hline $\begin{array}{l}\text { Polyester toddler wrap } \\
\text { (knit) }\end{array}$ & 17.50 & & & & & \\
\hline Silk napkin (woven) & 4.77 & & & & & \\
\hline $\begin{array}{l}\text { Nylon exercise pants } \\
\text { (woven) }\end{array}$ & 23.33 & & & & & \\
\hline Paper towel (bonded) & 10.41 & & & & & \\
\hline Tissue paper (bonded) & 20.2 & & & & & \\
\hline Copy paper (bonded) & 99.85 & & & & & \\
\hline Coffee filter, 2 layers & 14 & \multirow{5}{*}{$\begin{array}{l}85 \\
\mathrm{~L} / \mathrm{min}\end{array}$} & \multirow[t]{5}{*}{$\mathrm{NaCl}$ NIOSH method } & \multirow[t]{5}{*}{$100 \mathrm{~nm}$} & \multirow{5}{*}{$\begin{array}{l}\text { Solid } \\
\text { (Pei et al. 2020) }\end{array}$} & \multirow[t]{5}{*}{ No } \\
\hline Kitchen towel, 5 layers & 40 & & & & & \\
\hline Bed sheet, 5 layers & 54 & & & & & \\
\hline T-shirt, 5 layers & 64 & & & & & \\
\hline Shop towel, 5 layers & 69 & & & & & \\
\hline 4-ply tissue paper & 30.4 & \multirow[t]{3}{*}{ Laminar airflow } & \multirow{3}{*}{$\begin{array}{l}\mathrm{NaCl} \\
\text { ASTM method }\end{array}$} & \multirow[t]{3}{*}{$100 \mathrm{~nm}$} & \multirow{3}{*}{$\begin{array}{l}\text { Solid } \\
\text { (Li et al. 2020a) }\end{array}$} & \multirow[t]{3}{*}{ No } \\
\hline $\begin{array}{l}\text { 4-ply tissue paper folded } \\
\text { once }\end{array}$ & 41.2 & & & & & \\
\hline $\begin{array}{l}\text { Tissue paper }+ \text { kitchen } \\
\text { towel }\end{array}$ & 71.5 & & & & & \\
\hline
\end{tabular}


Table 3 (continued)

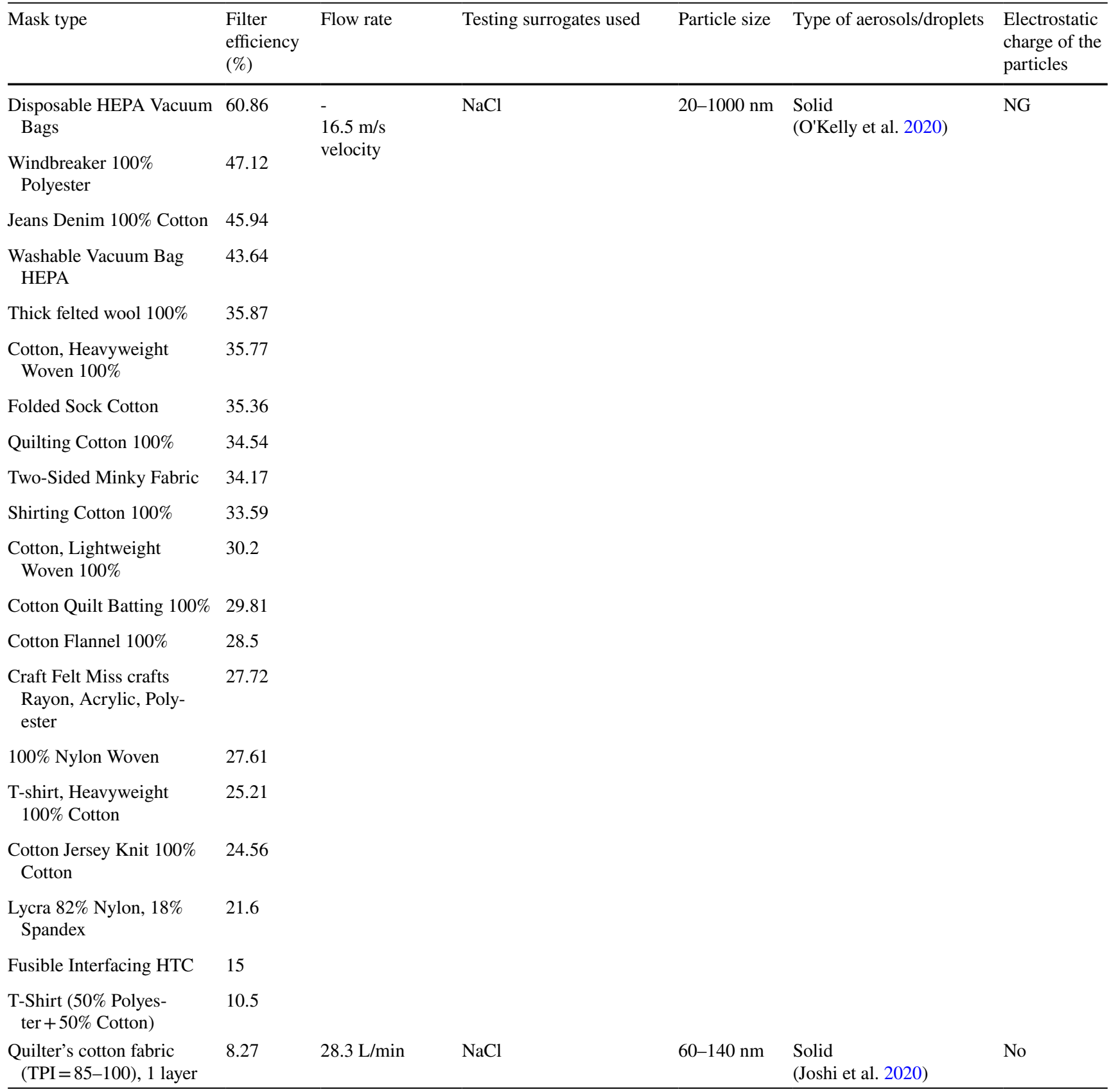


Table 3 (continued)

\begin{tabular}{|c|c|c|c|c|c|c|}
\hline Mask type & $\begin{array}{l}\text { Filter } \\
\text { efficiency } \\
(\%)\end{array}$ & Flow rate & Testing surrogates used & Particle size & Type of aerosols/droplets & $\begin{array}{l}\text { Electrostatic } \\
\text { charge of the } \\
\text { particles }\end{array}$ \\
\hline T-shirt $100 \%$ cotton & $12 \%$ & $30 \mathrm{~L} / \mathrm{min}$ & $\mathrm{NaCl}$ & $75 \mathrm{~nm}$ & \multirow{15}{*}{$\begin{array}{l}\text { Semi-solid } \\
\text { (Wang et al. 2020) }\end{array}$} & \multirow[t]{15}{*}{ NG } \\
\hline $\begin{array}{l}\text { Fleece sweater } 100 \% \\
\text { cotton }\end{array}$ & $6 \%$ & & & & & \\
\hline $\begin{array}{l}\text { Hairy tea towel } 80 \% \text { poly- } \\
\text { ester/20\% nylon }\end{array}$ & $23 \%$ & & & & & \\
\hline $\begin{array}{l}\text { Hairy tea towel + Fleece } \\
\text { sweater }\end{array}$ & $56 \%$ & & & & & \\
\hline Fleece sweater $+\mathrm{T}$-shirt & $12 \%$ & & & & & \\
\hline Fleece sweater, 2 layers & $11 \%$ & & & & & \\
\hline $\begin{array}{l}\text { Granular tea towel } 80 \% \\
\text { polyester/20\% nylon }\end{array}$ & $12 \%$ & & & & & \\
\hline $\begin{array}{l}\text { Fleece sweater }+ \text { Granular } \\
\text { tea towel }\end{array}$ & $11 \%$ & & & & & \\
\hline $\begin{array}{l}\text { Non-woven shopping bag } \\
100 \% \text { polypropylene }\end{array}$ & $14 \%$ & & & & & \\
\hline $\begin{array}{l}\text { Non-woven shopping } \\
\text { bag + T-shirt }\end{array}$ & $30 \%$ & & & & & \\
\hline $\begin{array}{l}\text { Non-woven shopping } \\
\text { bag + Hairy tea towel }\end{array}$ & $46 \%$ & & & & & \\
\hline $\begin{array}{l}\text { Non-woven shopping } \\
\text { bag + Granular tea towel }\end{array}$ & $47 \%$ & & & & & \\
\hline $\begin{array}{l}\text { Non-woven shopping } \\
\text { bag + Fleece sweater }\end{array}$ & $35 \%$ & & & & & \\
\hline $\begin{array}{l}\text { Non-woven shopping bag, } \\
2 \text { layers }\end{array}$ & $18 \%$ & & & & & \\
\hline $\begin{array}{l}\text { Pillowcase } 80 \mathrm{~s} \times 60 \mathrm{~s} \mathrm{Jet} \\
\text { satin }\end{array}$ & $0 \%$ & & & & & \\
\hline
\end{tabular}


Table 3 (continued)

\begin{tabular}{|c|c|c|c|c|c|c|}
\hline Mask type & $\begin{array}{l}\text { Filter } \\
\text { efficiency } \\
(\%)\end{array}$ & Flow rate & Testing surrogates used & Particle size & Type of aerosols/droplets & $\begin{array}{l}\text { Electrostatic } \\
\text { charge of the } \\
\text { particles }\end{array}$ \\
\hline $\begin{array}{c}1000 \text { TPI Bedsheet, } 1 \\
\text { layer (1000 TCBS1) }\end{array}$ & $48.95 \%$ & $3.0 \mathrm{~L} / \mathrm{min}$ & $\mathrm{NaCl}$ & $80-90 \mathrm{~nm}$ & $\begin{array}{l}\text { Solid } \\
\text { (Guha et al., 2021) }\end{array}$ & No \\
\hline $\begin{array}{l}1000 \text { TPI Bedsheet, } 2 \text { lay- } \\
\text { ers (1000 TCBS1) }\end{array}$ & $53.34 \%$ & & & & & \\
\hline $\begin{array}{l}1000 \text { TPI Pillowcase } \\
(1000 \text { TCPC })\end{array}$ & $41.62 \%$ & & & & & \\
\hline \multicolumn{7}{|l|}{ Blue Jeans } \\
\hline $\begin{array}{l}\text { Microfiber pillowcase, } \\
\text { layer (Microfiber PC1) }\end{array}$ & $30.82 \%$ & & & & & \\
\hline Canvas dropcloth & $18.89 \%$ & & & & & \\
\hline Silk Pillowcase & $12.90 \%$ & & & & & \\
\hline 200 TPI Pillowcase & $9.94 \%$ & & & & & \\
\hline 600 TPI Bedsheet 100 & $8.70 \%$ & & & & & \\
\hline Wash cloth & $7.89 \%$ & & & & & \\
\hline Flannel Bedsheets & $7.32 \%$ & & & & & \\
\hline $\begin{array}{l}\text { Microfiber Pillowcase, } 2 \\
\text { layers }\end{array}$ & $7.12 \%$ & & & & & \\
\hline Neck tube & $7.10 \%$ & & & & & \\
\hline Face tissue paper & $4.57 \%$ & & & & & \\
\hline Scarf & $3.79 \%$ & & & & & \\
\hline T-shirt & $3.68 \%$ & & & & & \\
\hline \multicolumn{7}{|l|}{ Paper towel } \\
\hline Cooling scarf & $2.94 \%$ & & & & & \\
\hline Bandana & $1.52 \%$ & & & & & \\
\hline 2-ply, $100 \%$ cotton mask & 77 & - & Cough particles & $10-1000 \mathrm{~nm}$ & $\begin{array}{l}\text { Liquid } \\
\text { (Li et al. 2020b) }\end{array}$ & NG \\
\hline 3-ply, cotton face mask & $\approx 28 \%$ & $28.3 \mathrm{~L} / \mathrm{min}$ & $\begin{array}{l}\text { KCL + sodium fluorescein } \\
\text { NIOSH modified method }\end{array}$ & $<600 \mathrm{~nm}$ & $\begin{array}{l}\text { Solid } \\
\text { (Lindsley et al. 2021) }\end{array}$ & $\mathrm{NO}$ \\
\hline $\begin{array}{l}\text { Cloth mask } 1 \text { (exhalation } \\
\text { valve) }\end{array}$ & $\approx 90 \%$ & $8 \mathrm{~L} / \mathrm{min}$ & PSL & $100 \mathrm{~nm}$ & $\begin{array}{l}\text { Solid } \\
\text { (Shakya et al. 2017) }\end{array}$ & NG \\
\hline Cloth mask 2 & $\approx 65 \%$ & & & & & \\
\hline Cloth mask 3 & $\approx 60 \%$ & & & & & \\
\hline $\begin{array}{l}\text { Cloth mask } 1 \text { (exhalation } \\
\text { valve) }\end{array}$ & $\approx 90 \%$ & $19 \mathrm{~L} / \mathrm{min}$ & & & & \\
\hline Cloth mask 2 & $\approx 32 \%$ & & & & & \\
\hline Cloth mask 3 & $\approx 28 \%$ & & & & & \\
\hline
\end{tabular}

$N G$ not given

\section{Penetration (PN)}

The percent aerosol penetration $(\mathrm{P})$ is defined as the ratio of the viral aerosols after filtration by maks (B) to the challenge aerosol concentration (A). Therefore, it will be calculated by this formula: $\frac{A}{B} \times 100$ (Tcharkhtchi et al. 2021). Four studies investigated the PN of particles from fabrics (Cherrie et al. 2018; Jung et al. 2013; Rengasamy et al. 2010; Shakya et al. 2017). Three of them used the hot plate method to fix the masks (Cherrie et al. 2018; Jung et al. 2013; Rengasamy et al. 2010), but one of them used maniquine based method 
Fig. 2 Choosing the best fabrics after FE, PN, FF, Q, and $\Delta \mathrm{P}$ filtrations

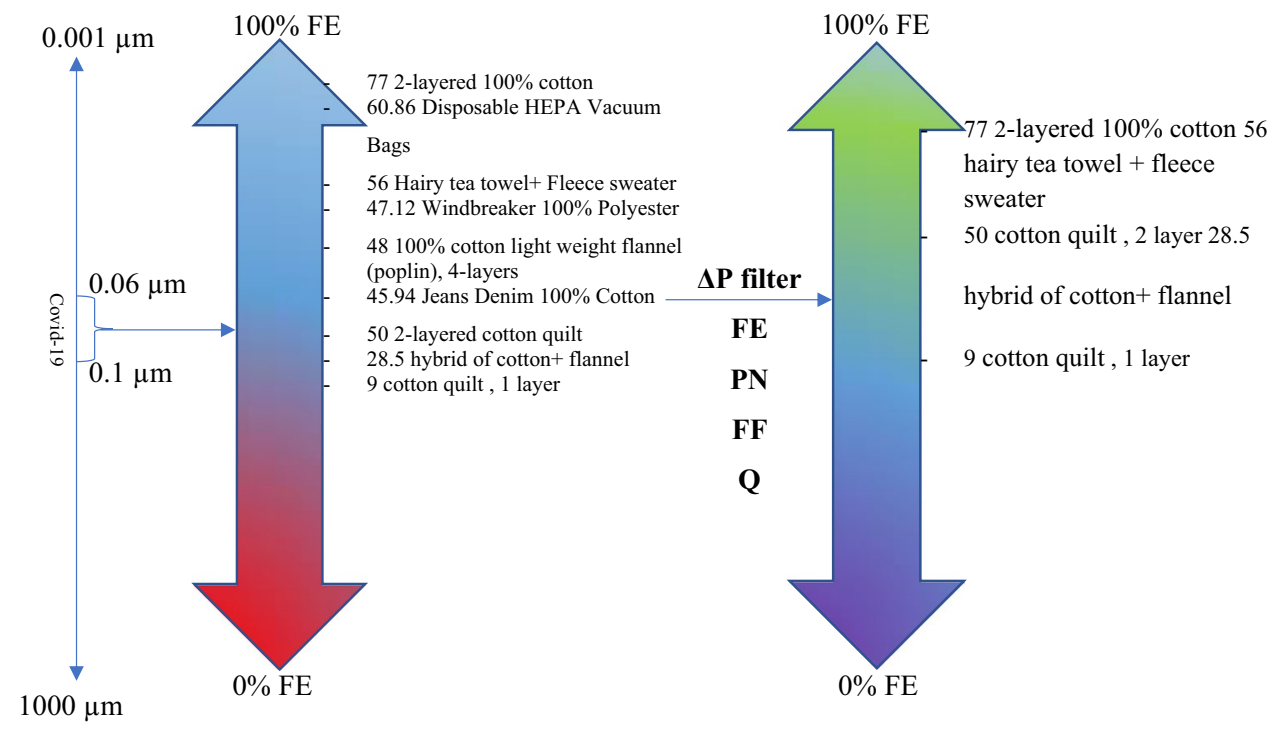

and for mask sealing used parafilm (Shakya et al. 2017). One study tested the PN of cotton and gauze handkerchiefs with the NIOSH and KFDA methods. First, they found there was no significant difference between these two methods; second, they reported that handkerchiefs, regardless of material, had no protection against $0.075 \mu \mathrm{m} \mathrm{NaCl}$ and paraffin oil particles (PN > 98\%) (Jung et al. 2013). Another study compared the PN of three commercial cloth masks. The one with an exhaust valve and a cone or tetrahedral shape that can fit well to the face had the least PN in both flow rates $\left(8 \& 19\right.$ L.min ${ }^{-1}$ ) (Shakya et al. 2017). The improved performance with well-fitting masks suggests that leakage may be an issue in studies that utilize mannequins to test for filter penetration. Rengasamy et al. showed variable PN rates in cloth masks and fabric materials. The cloth masks PN was between 50 and $90 \%$ for polydisperse and 70-80\% for $100 \mathrm{~nm}$ monodisperse aerosols at $33 \mathrm{~L} \cdot \mathrm{min}^{-1}$. The PN of fabric materials for polydisperse aerosols was between $40-89 \%$, and for monodisperse ones was among $9-95 \%$ at $33 \mathrm{~L} \cdot \mathrm{min}^{-1}$ indicating that all of them had marginal efficacy (Rengasamy et al. 2010). The last study found that by increasing the flow rate, PN increased. In this study, PN was between 0.2 and $20.7 \%$. The lowest value reported was for the "Yimeijian" mask, and the highest was for the "Gucheng" mask (Cherrie et al. 2018).

\section{Pressure drop (breathability)}

Seventeen studies evaluated pressure drop $(\Delta \mathrm{P})$ (Aydin et al. 2020; Davies et al. 2013; Drewnick et al. 2021; Hao et al. 2020; Joshi et al. 2020; Jung et al. 2013; Konda et al. 2020a; Long et al. 2020; Maher et al. 2020; Park and Jayaraman 2020; Pei et al. 2020; Teesing et al. 2020; Varallyay et al. 2020; Wang et al. 2020; Zangmeister et al. 2020; Zhao et al. 2020; Guha et al. 2021), which indicated breathability or comfort when you are breathing and face fitness of the mask or presence any leakage. $\Delta \mathrm{P}$ has a reverse relation with breathability, which means by increasing the $\Delta \mathrm{P}$, breathability decreases. Also, in some studies, $\Delta \mathrm{P}$ significantly improved by increasing the layers (Aydin et al. 2020; Davies et al. 2013; Jung et al. 2013; Wang et al. 2020). In one study, however, with increasing the layers of tightly woven fabrics, $\Delta \mathrm{P}$ significantly declined (Guha et al. 2021). Additionally, breathability depends strongly on porosity and TPI, which means increasing the porosity increases breathability, but increased TPI has the opposite effect. (Aydin et al. 2020; Zhao et al. 2020).

Among different materials: cotton (Long et al. 2020; Maher et al. 2020), cotton-quilt (Konda et al. 2020a; Teesing et al. 2020), cotton bandana (Hao et al. 2020), cotton block hand towel (Zangmeister et al. 2020), pillowcase 100\% woven cotton (Davies et al. 2013; Varallyay et al. 2020; Zhao et al. 2020), 100\% cotton T-shirt (Davies et al. 2013; Varallyay et al. 2020), gauze and cotton handkerchiefs (Jung et al. 2013), fleece sweater (Wang et al. 2020), woven 100\% silk scarf and thick fleece-Knitted $100 \%$ polyester (Varallyay et al. 2020), 100\% polyester (Cooling scarf) and 100\% microfiber polyester (bandana mask) (Guha et al. 2021), and muslin (Drewnick et al. 2021) were most breathable fabrics.

The materials with the least breathability were vacuum cleaner bag and tea towel because of their thickness and stiffness (Davies et al. 2013; Long et al. 2020; Maher et al. 2020), non-woven shopping bag + T-shirt (Wang et al. 2020), microfiber cloth $-80 \%$ polyester-20\% polyamide (TPI: 38 ) (Varallyay et al. 2020), leather (Teesing et al. 2020), cellulose copy paper and nylon (Zhao et al. 2020), coffee filter (Hao et al. 2020), plain polyester (Zangmeister et al. 2020), 5-layer bedsheet (Pei et al. 2020), poplin (Drewnick et al. 
2021), one and two layers of One Thousand TPI $100 \%$ cotton Bedsheet (Guha et al. 2021), and chiffon (Konda et al. 2020a).

One study measured breathability $(\beta)$, which is related to both the pressure drop $(\Delta \mathrm{P})$ and the changes in the flow rate, then reported that, for the same porosity, knit fabrics had higher breathability than woven fabrics (Aydin et al. 2020). Loosely knit or woven fabrics in another study considered highly breathable compared to tightly woven fabrics, which were less breathable (Guha et al. 2021). Furthermore, used knitted undershirt ( $75 \%$ cotton-25\% polyester) showed the most breathability but, used knitted shirt (100\% cotton) and used woven shirt $(70 \% \mathrm{C}-30 \% \mathrm{PE})$ were the least breathable fabrics. It has been shown that using cotton fabrics that have been washed experience shrinkage that results in pore size decrease and less breathability. Also, if various cleaning products (e.g., starch) are used for washing cloth fabrics, they can alter breathability (Aydin et al. 2020). Albeit, we have to keep in mind that cloth masks reuse will increase the risk of infection unless washing properly (Szarpak et al. 2020).

Additionally, one study tested all the fabrics after one cycle of washing with a home laundry machine. In this study, dampness has been tested. Some fabrics FE like quilting cotton, cotton flannel after dampesss has not been changed. However, some of them like denim FE substantially decreased (O'Kelly et al. 2020). Therefore, we can conclude that washing can affect some fabrics FE but not all. Another study reported no significant pressure drop indicated between different fabrics (Park and Jayaraman 2020).

\section{Filter quality (Q)}

Four studies evaluated the filter quality of different fabrics (Drewnick et al. 2021; Hao et al. 2020; Zangmeister et al. 2020; Zhao et al. 2020). Filter quality is a factor for indicating filter performance. It is related to two factors: FE and pressure drop; by increasing the FE and decreasing the pressure drop, the filter's quality increases. It will also not be affected by the number of layers of a single-layer fabric (Zangmeister et al. 2020; Zhao et al. 2020). Furthermore, a study found no correlation between filter quality and TPI (Drewnick et al. 2021). In one study, cotton sweaters and T-shirts had better filter quality, but cellulose copy paper had the worst quality (Zhao et al. 2020). The second study reported better filter quality for vacuum bags (Drewnick et al. 2021; Hao et al. 2020), and the coffee filter had the lowest quality (Hao et al. 2020). In the third study, cotton hand towels had better filter quality, and plain polyester had a low filter quality (Zangmeister et al. 2020). In the fourth study, silk had the least quality (Drewnick et al. 2021).

\section{Fit factor (FF)}

FF describes the penetration around the mask and towards the breathing zone and expresses how good the fit of a mask is on the face. FF is the ratio of time-averaged particle concentration outside and inside mask (van der Sande et al. 2008). The FE of a mask is dependent on the FF, while the FF itself could be influenced by some factors such as the type of user's activity and facial characteristics (Pacitto et al. 2019). One study done by Clapp et al. measured the fitted filtration efficiency (FFE) ranged from 26.5 to $79 \%$ by OSHA regulations. All the samples were fitted on a man face with no beard in different ways (Clapp et al. 2020).

Additionally, Teesing et al. considered a FF of 100 or higher as a good fit. In their study, none of the cotton masks report a well fit (Teesing et al. 2020). Protection factors (PF) is a similar concept to FF that is related to Portacount devices, but FF is used by OSHA (van der Sande et al. 2008). Mueller et al. found that surgical-type cloth masks had less FE because of their poor fit. Therefore, adding up a nylon layer to the cloth masks decreased gaps and increased FE (Mueller et al. 2020). Davis et al. revealed that stretchy fabrics like $100 \%$ cotton T-shirts are more fittable and preferable than non-stretch fabrics with the same FE (Davies et al. 2013). Lindsley et al. analyzed the FF of 3-ply cotton face mask which was 1.3 and showed the $50.9 \%$ FE (Lindsley et al. 2021).

\section{Discussion}

Currently, many studies have been evaluated on fabric masks, but none of them have compared the protected efficacy of fabric masks. This issue has become even more complex when one compares different types of fabrics, different layers of fabrics. In this systematic review, we attempt to compare fabric masks based on filtration efficiency, pressure drop, QF, penetration, and fit factor. different fabric masks' performance to find the best potential choice to limit the spread of respiratory particles. In two studies, singlelayered cotton quilt (TPI $\approx 80$ ) showed $\mathrm{FE} \approx 9 \%$ (Joshi et al. 2020; Konda et al. 2020a). After adding another layer of the cotton quilt, its efficiency increased five times $(\mathrm{FE}=50 \%)$. Also quilting cotton was one of the best fabrics as it showed an acceptable FE for both damp and dry particles and good breathability (O'Kelly et al. 2020). Moreover, by increasing the cotton quilt's TPI to 120 , its efficiency increased by more than ten times (FE $=96 \%$ ) (Joshi et al. 2020). Despite increasing the number of layers or increasing the TPI of the fabric, all samples were breathable, and that was a good point. The tighter a fabric's weave, the smaller the pores and the increase in FE as 1000 TPI $100 \%$ cotton bed sheets showed modest FE (48.9\%) but high $\Delta \mathrm{P}$, which exceeded 
the limit (Guha et al. 2021). In contrast, a higher yarn count and a looser weave resulted in a lower FE (Zangmeister et al. 2020). Cotton with a higher yarn count and a looser weave showed a lower FE. Perhaps the higher yarn count causes more penetration and less FE. It should be mentioned that the best performing cloth materials have moderate yarn counts (Zangmeister et al. 2020). In addition to the cotton quilt, other $100 \%$ cotton fabrics like cotton flannel revealed well FE and tolerable $\Delta \mathrm{P}$ (O'Kelly et al. 2020). Additionally, in the Zangmeister et al. study, 2-layers $100 \%$ cotton fabrics (TPI $=100=150)$ had $24 \% \leq \mathrm{FE} \leq 32 \%$. In this study, 4-layer $100 \%$ cotton light-weight flannel (poplin) had elevated FE to $48 \%$ compared with two layers $(\mathrm{FE}=24.3 \%)$. After increasing the layers, $\Delta \mathrm{P}$ increased, and filter quality decreased (Zangmeister et al. 2020). Hence, $100 \%$ cotton fabric like cotton flannel with one to two layers can be a good option. Also, in the Li et al. study, a 2-ply 100\% cotton fabric's FE was $77 \%$ but, they reported that all tested cloth masks had less FE for particles $<1000 \mathrm{~nm}$ ( $\mathrm{Li}$ et al. 2020b). We note that the efficacy of these two is different, perhaps because of the difference in the particle size range, which was greater in the Li et al. study (50-825 nm vs 10-1000 nm). To enable a better comparison, 2-layered $100 \%$ cotton should be tested in the same situation. Given this study and the previous one, it can be concluded this fabric can be a good choice. A 3-ply cotton face mask showed about 28\% FE for particles $<600 \mathrm{~nm}$ (Lindsley et al. 2021). In Zhao et al.'s study, the copy paper, while showing high FE, also had a very high $\Delta \mathrm{P}$ that made it of low quality. Despite having good filtration, copy paper is not a good choice for a mask (Zhao et al. 2020). O'Kelly et al., after evaluating different fabrics, stated that vacuum cleaner bags had the best efficacy. Also, Windbreaker 100\% Polyester and Jeans Denim 100\% Cotton had good FE, but they were not as breathable as vacuum cleaner bags (O'Kelly et al. 2020). Therefore, a vacuum cleaner HEPA bag seems a good choice as a filter layer in a cloth mask but, three studies reported it as an unbreathable fabric (Davies et al. 2013; Long et al. 2020; Maher et al. 2020). This discrepancy refers to the other materials tested in O'kellys study. As we mentioned, the vacuum cleaner bag is more breathable than jeans and a windbreakerFabrics like silk have enhanced FE because of their electrostatic properties that attract and hold particles. This is an important point that is being considered in mask design (Zhao et al. 2020). In another study, hairy tea towels alone had 23\% efficacy. After it was combined with the fleece sweater, its efficacy converted to the best among other materials and became more than $50 \%$. Its $\Delta \mathrm{P}$ was under $49 \mathrm{~Pa}$, which shows it is a breathable fabric. Fleece sweater is one of the most breathable fabrics that its FE is reported 6\%. (Wang et al. 2020). Therefore, it is a good choice for combining with other fabrics to make a breathable and more effective mask. Pei et al. evaluated different fabrics against particles in the
30-1000 nm size range. For $100 \mathrm{~nm}$ particles, a 5-layer shop towel had the best efficacy, but the study did not report its explicit material. Furthermore, the figure of merit related to $\mathrm{FE}$ and $\Delta \mathrm{P}$ did not compare the shop towel with the other materials (Pei et al. 2020). So, we cannot decide if it is a reasonable choice. In the Li et al. study, one layer of 4-ply tissue paper followed by two layers of kitchen towel showed the best efficacy $(\mathrm{FE}=71.5 \%$ for $100 \mathrm{~nm}$ particles). They also reported that the most particle penetrating size was between 100 and $125 \mathrm{~nm}$ (Li et al. 2020a). Although the mask has the least efficacy at $100 \mathrm{~nm}$, it is suitable for preparing fabric masks. However, it should be noted the mask breathability and quality factor were not reported and require further study. Considering these results, hybrid fabrics can work well as a mask. In the second step, we are going to discuss cloth mask studies. Shakya et al. compared three different cloth masks but they did not mention the cloth mask fabric materials. They recently reported the cloth mask with an exhalation valve had better filtration effectiveness and less particle penetration (Shakya et al. 2017). Thus, we are unable to fully evaluate their findings. In addition to the FE, some studies compared different cloth masks by using penetration rates. Three did not mention the details about cloth mask materials (Cherrie et al. 2018; Rengasamy et al. 2010; Shakya et al. 2017). In addition to the cloth masks, Rengasamy et al. had also examined several different fabrics: three brands of Sweatshirt, T-shirt, towel, and scarf in different materials. Penetration for mono and polydisperse aerosols was variable, and it showed marginal efficacy for these materials, especially for particles $<1000 \mathrm{~nm}$ (Rengasamy et al. 2010). Jung et al. investigated the penetration of cotton and gauze handkerchiefs with two KFDA and NIOSH methods. This evaluation showed that both cotton and gauze handkerchiefs had more than $98 \%$ penetration, and after folding, penetration decreased to $87 \%$, which is still high. These results show us that handkerchiefs are not able to filter $75 \mathrm{~nm}$ particles well (Jung et al. 2013). Fabrics tested in penetration studies could not filter particles well. Between two RCTs tested on health care workers (HCWs), the first RCT reported that cotton yarn masks were not recommended for HCWs. The cloth mask layer count was not mentioned in this study (Yang et al. 2011). The second one used a 2-layered cotton cloth mask, but the highest rate of respiratory infections was in HCWs who wore cloth masks (MacIntyre et al. 2015b). In both studies, only one type of cloth mask was used, not different types. We recommend more clinical trials to compare cloth masks but not for HCWs, as two RCTs reported them insufficient. Another clinical study reported that a 3 -layered $100 \%$ cotton mask had no significant difference from a surgical mask but did not report its exact FE (Ho et al. 2020). So, we are not able to report it as a good choice. Although our study aimed to compare just cloth masks FE. Masks of category 1 which 
filters $>95 \%$ of particles $>3 \mu \mathrm{m}$ (respirators), and medical masks are more effective than cloth masks (Chughtai et al. 2020) as some countries like France banned the use of cloth masks with the outburst of new covid-19 variants (Mahase 2021).

\section{Limitations}

Our results and conclusion are based on all the studies that are done up to the present time, which are mostly experimental. Thus, there is a great need for clinical trials. In these studies, instead of using the Covid-19 particles, different surrogates (sodium chloride, cough particles, $\mathrm{KCL}+$ sodium fluorescein, PSL) have been used. All the studies that analyzed the FE of masks against particles in the Covid-19 particle size range with the use of different surrogates did not evaluate the FF. Because of that, we could not compare masks in this field. At the end of this study, we bring some tables that compare studies in different aspects. But because of the different situations of these studies, we could not bring a complete comparison, and some factors like the type of the surrogates and flow rate that have critical effects of FE are not mentioned.

\section{Conclusion}

Cloth masks and fabrics have provided some protection, with some variability noted. The use of cloth masks by the general population can protect them to some degree. The purpose of this study is to find the best fabrics, especially against Covid-19. We compare different materials for their filtration, efficacy, penetration, pressure drop, and filter quality. The best performing fabrics are: cotton quilt (1-2 layers), cotton flannel, 2-layered 100\% cotton, hybrid of cotton + flannel, and hairy tea towel + fleece sweater. Multi-layered fabrics showed better filtrationefficacy, and breathability. One RCT reported a 3-layered 100 cotton cloth mask had equal efficacy with a surgical mask. According to two RCTs, cloth mask use is not recommended for HCWs. At the end, we have to mention that limited clinical trials showed the cloth masks or fabrics effectiveness in Covid-19; these findings are our suggestion after reviewing all articles in this area. So the use of these types of masks may not be appropriate for Covid 19 .

\section{Acknowledgements Not applicable}

Author contribution MA, FMS, SN, MA, and OM contributed to conception, design, and preparation of the manuscript. MA, SN conducted literature search and contributed in article selection, MA and $\mathrm{SN}$ made data extraction. MA, SN, and OM contributed to acquisition, and interpretation. MA, FMS, SN, MA, and OM made substantial contributions in drafting the manuscript and revising it critically for important intellectual content. All authors have read and approved the final version of manuscript.

Data availability The datasets are available from the corresponding author on formal and logic request.

\section{Declarations}

Ethics approval and consent to participate Not applicable.

Consent for publication Not applicable.

Competing interests The authors declare no competing interests.

\section{References}

Anonymous World Health Organization available at: https://covid19. who.int/ data access November 2020

Asadi S, Cappa CD, Barreda S, Wexler AS, Bouvier NM, Ristenpart WD (2020) Efficacy of masks and face coverings in controlling outward aerosol particle emission from expiratory activities. Scientific Reports 10

Aydin O, Emon B, Cheng S, Hong L, Chamorro LP, Saif MTA (2020) Performance of fabrics for home-made masks against the spread of COVID-19 through droplets: A quantitative mechanistic study. Extreme Mechanics Letters 40

Bhattacharjee S, Bahl P, Chughtai AA, Mac-Intyre CR (2020) Lastresort strategies during mask shortages: optimal design features of cloth masks and decontamination of disposable masks during the COVID-19 pandemic. BMJ Open Respir Res 7:e000698

Bowen LE (2010) Does that face mask really protect you? Appl Biosaf 15:67-71

Carr IA, Hariharan P, Guha S (2020) Letter to the Editor Regarding Aerosol Filtration Efficiency of Common Fabrics Used in Respiratory Cloth Masks. ACS Nano 14(9):10754-10755

Chen HL, Chung SH, Jhuo ML (2013) Efficiency of different respiratory protective devices for removal of particulate and gaseous reactive oxygen species from welding fumes. Arch Environ Occup Health 68:101-106

Cherrie JW, Apsley A, Cowie H, Steinle S, Mueller W, Lin C, Horwell CJ, Sleeuwenhoek A, Loh M (2018) Effectiveness of face masks used to protect Beijing residents against particulate air pollution. Occup Environ Med 75:446-452

Chinazzi M, Davis JT, Ajelli M, Gioannini C, Litvinova M, Merler S, y Piontti AP, Mu K, Rossi L, Sun K (2020) The effect of travel restrictions on the spread of the 2019 novel coronavirus (COVID19) outbreak. Science 368:395-400

Chughtai AA, Seale H, Macintyre CR (2020) Effectiveness of cloth masks for protection against severe acute respiratory syndrome coronavirus 2. Emerg Infect Dis 26(10):e200948

Clapp PW, Sickbert-Bennett EE, Samet JM, Berntsen J, Zeman KL, Anderson DJ, Weber DJ, Bennett WD (2020) Evaluation of Cloth Masks and Modified Procedure Masks as Personal Protective Equipment for the Public during the COVID-19 Pandemic. JAMA Intern Med 181:463-469

Clase CM, Fu EL, Ashur A, Beale RC, Clase IA, Dolovich MB, Jardine MJ, Joseph M, Kansiime G, Mann JF (2020) Forgotten Technology in the COVID-19 Pandemic. Filtration Properties of Cloth and Cloth Masks: A Narrative Review, Mayo Clinic Proceedings. Elsevier 
Davies A, Thompson KA, Giri K, Kafatos G, Walker J, Bennett A (2013) Testing the Efficacy of Homemade Masks: Would They Protect in an Influenza Pandemic? Disaster Med Public Health Prep 7:413-418

Drewnick F, Pikmann J, Fachinger F, Moormann L, Sprang F, Borrmann S (2021) Aerosol filtration efficiency of household materials for homemade face masks: Influence of material properties, particle size, particle electrical charge, face velocity, and leaks. Aerosol Sci Technol 55:63-79

Fischer EP, Fischer MC, Grass D, Henrion I, Warren WS, Westman E (2020) Low-cost measurement of face mask efficacy for filtering expelled droplets during speech. Sci Adv 6(36):eabd3083

Godoy LRG, Jones AE, Anderson TN, Fisher CL, Seeley KM, Beeson EA, Zane HK, Peterson JW, Sullivan PD (2020) Facial protection for healthcare workers during pandemics: a scoping review. BMJ Glob Health 5:e002553

Guha S, Herman A, Carr IA, Porter D, Natu R, Berman S, Myers MR (2021) Comprehensive characterization of protective face coverings made from household fabrics. PloS One 16(1):e0244626

Hao W, Parasch A, Williams S, Li J, Ma H, Burken J, Wang Y (2020) Filtration performances of non-medical materials as candidates for manufacturing facemasks and respirators. Int J Hyg Environ Health 229:113582

Ho KF, Lin LY, Weng SP, Chuang KJ (2020) Medical mask versus cotton mask for preventing respiratory droplet transmission in micro environments. Sci Total Environ 735:139510

Howard J, Huang A, Li Z, Tufekci Z, Zdimal V, van der Westhuizen H-M, von Delft A, Price A, Fridman L, Tang L-H (2020) Face masks against COVID-19: an evidence review 118(4):e2014564118

Joshi M, Khan A, Sapra BK (2020) Quick laboratory methodology for determining the particle filtration efficiency of face masks/ respirators in the wake of COVID-19 pandemic. J Ind Textiles

Jung H, Kim JK, Lee S, Lee J, Kim J, Tsai P, Yoon C (2013) Comparison of filtration efficiency and pressure drop in anti-yellow sand masks, quarantine masks, medical masks, general masks, and handkerchiefs. Aerosol Air Qual Res 14:991-1002

Konda A, Prakash A, Moss GA, Schmoldt M, Grant GD, Guha S (2020a) Aerosol Filtration Efficiency of Common Fabrics Used in Respiratory Cloth Masks. ACS Nano 14:6339-6347

Konda A, Prakash A, Moss G, Schmoldt M, Grant G, Guha S (2020b) Correction to aerosol filtration efficiency of common fabrics used in respiratory cloth masks. ACS Nano 14(8):10742-10743

Lake MA (2020) What we know so far: COVID-19 current clinical knowledge and research. Clin Med 20:124

Li IW, Fan JK, Lai AC, Lo CM (2020a) Home-made masks with filtration efficiency for nano-aerosols for community mitigation of COVID-19 pandemic. Public Health 188:42-50

Li L, Niu M, Zhu Y (2020b) Assessing the effectiveness of using various face coverings to mitigate the transport of airborne particles produced by coughing indoors. Aerosol Sci Technol

Lindsley WG, Blachere FM, Law BF, Beezhold DH, Noti JD (2021) Efficacy of face masks, neck gaiters and face shields for reducing the expulsion of simulated cough-generated aerosols. Aerosol Sci Technol 55:449-457

Liu Z, Yu D, Ge Y, Wang L, Zhang J, Li H, Liu F, Zhai Z (2019) Understanding the factors involved in determining the bioburdens of surgical masks. Ann Transl Med 7(23):754

Long KD, Woodburn EV, Berg IC, Chen V, Scott WS (2020) Measurement of filtration efficiencies of healthcare and consumer materials using modified respirator fit tester setup. PLoS ONE 15

Lu H, Yao D, Yip J, Kan CW, Guo H (2020) Addressing COVID-19 spread: Development of reliable testing system for mask reuse. Aerosol Air Qual Res 20:2309-2317
Lustig SR, Biswakarma JJH, Rana D, Tilford SH, Hu W, Su M, Rosenblatt MS (2020) Effectiveness of Common Fabrics to Block Aqueous Aerosols of Virus-like Nanoparticles. ACS Nano 14:7651-7658

Ma QX, Shan H, Zhang HL, Li GM, Yang RM, Chen JM (2020) Potential utilities of mask-wearing and instant hand hygiene for fighting SARS-CoV-2. J Med Virol 92:1567-1571

MacIntyre CR, Seale H, Dung TC, Hien NT, Nga PT, Chughtai AA, Rahman B, Dwyer DE, Wang Q (2015) A cluster randomised trial of cloth masks compared with medical masks in healthcare workers. BMJ Open 5:e006577

MacIntyre CR, Seale H, Dung TC, Hien NT, Nga PT, Chughtai AA, Rahman B, Dwyer DE, Wang QY (2015b) A cluster randomised trial of cloth masks compared with medical masks in healthcare workers. BMJ Open 5(4):e006577

Mahase E (2021) Covid-19: are cloth masks still effective? And other questions answered. BMJ 372:n432

Maher B, Chavez R, Tomaz GCQ, Nguyen T, Hassan Y (2020) A fluid mechanics explanation of the effectiveness of common materials for respiratory masks. Int J Infect Dis 99:505-513

Moher D, Shamseer L, Clarke M, Ghersi D, Liberati A, Petticrew M, Shekelle P, Stewart LA (2015) Preferred reporting items for systematic review and meta-analysis protocols (PRISMA-P) 2015 statement. Syst Rev 4:1

Mueller AV, Eden MJ, Oakes JM, Bellini C, Fernandez LA (2020) Quantitative Method for Comparative Assessment of Particle Removal Efficiency of Fabric Masks as Alternatives to Standard Surgical Masks for PPE. Matter 3:950-962

Neupane BB, Mainali S, Sharma A, Giri B (2019) Optical microscopic study of surface morphology and filtering efficiency of face masks. PeerJ 7:e7142

O'Kelly E, Pirog S, Ward J, Clarkson PJ (2020) Ability of fabric face mask materials to filter ultrafine particles at coughing velocity. BMJ Open 10(9):e039424

Organization WH (2020a) Advice on the use of masks in the community, during home care, and in health care settings in the context of COVID-19: interim guidance, 19 March 2020, World Health Organization

Organization WH (2020b) Advice on the use of masks in the context of COVID-19: interim guidance, 5 June 2020, World Health Organization

Pacitto A, Amato F, Salmatonidis A, Moreno T, Alastuey A, Reche C, Buonanno G, Benito C, Querol X (2019) Effectiveness of commercial face masks to reduce personal PM exposure. Sci Total Environ 650:1582-1590

Park S, Jayaraman S (2020) From containment to harm reduction from SARS-CoV-2: a fabric mask for enhanced effectiveness, comfort, and compliance. J Textile Inst 112(7):1144-1158

Pei C, Ou Q, Kim SC, Chen SC, Pui DYH (2020) Alternative face masks made of common materials for general public: Fractional filtration efficiency and breathability perspective. Aerosol Air Qual Res 20:2581-2591

Rengasamy S, Eimer B, Shaffer RE (2010) Simple respiratory protection - Evaluation of the filtration performance of cloth masks and common fabric materials against 20-1000 nm size particles. Ann Occup Hyg 54:789-798

Rodriguez-Palacios A, Cominelli F, Basson AR, Pizarro TT, Ilic S (2020) Textile masks and surface covers-a spray simulation method and a "Universal Droplet Reduction Model" against respiratory pandemics. Front Med 7:260. https://doi.org/10. 3389/fmed.2020.00260

Santos M, Torres D, Cardoso PC, Pandis N, Flores-Mir C, Medeiros R, Normando AD (2020) Are cloth masks a substitute to medical masks in reducing transmission and contamination? A systematic review. Braz Oral Res 34 
Scells H, Zuccon G, Koopman B, Deacon A, Azzopardi L, Geva S (2017) Integrating the framing of clinical questions via PICO into the retrieval of medical literature for systematic reviews, Proceedings of the 2017 ACM on Conference on Information and Knowledge Management, pp. 2291-2294

Shakya KM, Noyes A, Kallin R, Peltier RE (2017) Evaluating the efficacy of cloth facemasks in reducing particulate matter exposure. J Expo Sci Environ Epidemiol 27:352-357

Sunjaya AP, Morawska L (2020) Evidence Review and Practice Recommendation on the Material, Design, and Maintenance of Cloth Masks. Disaster Medicine and Public Health Preparedness, $1-5$

Szarpak L, Smereka J, Filipiak KJ, Ladny JR, Jaguszewski M (2020) Cloth masks versus medical masks for COVID-19 protection. Cardiol J 27(2):218-219

Tcharkhtchi A, Abbasnezhad N, Zarbini Seydani M, Zirak N, Farzaneh S, Shirinbayan M (2021) An overview of filtration efficiency through the masks: Mechanisms of the aerosols penetration. Bioact Mater 6(1):106-122

Teesing GR, van Straten B, de Man P, Horeman-Franse T (2020) Is there an adequate alternative to commercially manufactured face masks? A comparison of various materials and forms. J Hosp Infect 106:246-253

Tufanaru C, Munn Z, Aromataris E, Campbell J, Hopp L (2020) Chapter 3: Systematic reviews of effectiveness. In: Aromataris E, Munn $\mathrm{Z}$ (Editors). JBI Manual for Evidence Synthesis. JBI. Available from https://synthesismanual.jbi.global. https://doi.org/10.46658/ JBIMES-20-04

Ueki H, Furusawa Y, Iwatsuki-Horimoto K, Imai M, Kabata H, Nishimura H, Kawaoka Y (2020) Effectiveness of face masks in preventing airborne transmission of SARS-CoV-2. mSphere 5(5): $000637-20$

van der Sande M, Teunis P, Sabel R (2008) Professional and homemade face masks reduce exposure to respiratory infections among the general population. PLoS One 3:e2618

Varallyay C, Li N, Case B, Wolf B (2020) Material suitability testing for non-medical grade community face masks to decrease viral transmission during a pandemic. Disaster Medicine and Public Health Preparedness 1-7. https://doi.org/10.1017/dmp.2020.262
Wang DJ, You YJ, Zhou XL, Zong ZY, Huang H, Zhang H, Yong X, Cheng YF, Yang L, Guo Q, Long YL, Liu Y, Huang J, Du L (2020) Selection of homemade mask materials for preventing transmission of COVID-19: a laboratory study. Plos One 15(10):e0240285

Wen Z, Lu J, Li J, Li N, Zhao J, Wang J, Yu L (2010) Yang W (2010): Determining the filtration efficiency of half-face medical protection mask (N99) against viral aerosol. Aerobiologia (bologna) 26(3):245-251

Whiley H, Keerthirathne TP, Nisar MA, White MAF, Ross KE (2020) Viral filtration efficiency of fabric masks compared with surgical and $\mathrm{n} 95$ masks. Pathogens 9:1-8

Wiersinga WJ, Rhodes A, Cheng AC, Peacock SJ, Prescott HC (2020) Pathophysiology, transmission, diagnosis, and treatment of coronavirus disease 2019 (COVID-19): a review. JAMA 324:782-793

Xiao L, Sakagami H, Miwa N (2020) A new method for testing filtration efficiency of mask materials under sneeze-like pressure. In Vivo 34:1637-1644

Yang P, Seale H, MacIntyre CR, Zhang H, Zhang Z, Zhang Y, Wang X, Li X, Pang X, Wang Q (2011) Mask-wearing and respiratory infection in healthcare workers in Beijing, China. Braz J Infect Dis 15:102-108

Zangmeister CD, Radney JG, Vicenzi EP, Weaver JL (2020) Filtration Efficiencies of Nanoscale Aerosol by Cloth Mask Materials Used to Slow the Spread of SARS-CoV-2. ACS Nano 14:9188-9200

Zhao M, Liao L, Xiao W, Yu X, Wang H, Wang Q, Lin YL, KilincBalci FS, Price A, Chu L, Chu MC, Chu S, Cui Y (2020) Household Materials Selection for Homemade Cloth Face Coverings and Their Filtration Efficiency Enhancement with Triboelectric Charging. Nano Lett 20:5544-5552

Publisher's note Springer Nature remains neutral with regard to jurisdictional claims in published maps and institutional affiliations. 